\title{
Evaluating Positive and Negative Effects of Seven Biogenic Metal-based Nanoparticles on Seed Germination and Seedling of Nano-primed Wheat and Flax Seeds
}

Maryam Bayat

RUDN University: Rossijskij universitet druzby narodov

Meisam Zargar ( $\square$ zargar_m@pfur.ru )

RUDN: Rossijskij universitet druzby narodov https://orcid.org/0000-0002-5208-0861

\section{Research Article}

Keywords: Metal-based nanoparticles, biosynthesis, wheat, flax, seed germination, seedling growth, phytotoxicity

Posted Date: November 19th, 2021

DOI: https://doi.org/10.21203/rs.3.rs-1007379/v1

License: (c) (i) This work is licensed under a Creative Commons Attribution 4.0 International License. Read Full License 


\section{Abstract}

Seed germination is the first and the most susceptible stage in plant's growing phases, so could be considered as an index to evaluate the effect of newly developed materials like nanoparticles (NPs), providing useful information for researchers. In our experiments, germination tests have been carried out in Petri plates, containing wet filter paper and nano-primed seeds. We had biosynthesized seven nanoparticles in our previous researches, including calcinated and non-calcinated zinc oxide, zinc, magnesium oxide, silver, copper and iron nanoparticles. The effect of these biogenic nanoparticles and their counterpart metallic salts including zinc acetate, magnesium sulphate, silver nitrate, copper sulphate and iron (III) chloride was studied on two popularly grown plants, wheat and flax, in laboratory condition to obtain preliminary information for future field experiments. Germination percentage, shoot length, root length, seedlings length, root-shoot ratio, seedling vigor index (SVI), shoot length stress tolerance index (SLSI) and root length stress tolerance index (RLSI) were calculated at $2^{\text {nd }}$ and $7^{\text {th }}$ days of the experiment. According to the results, the response of the plants to metal containing nanoparticles and metal salts mainly depend on type of the metal, plant species, concentration of the NP suspension or salt solution, condition of the exposure and the stage of growth.

\section{Introduction}

The rapidly growing population and crop consumption cause a high demand of using fertilizers, which are critical for plant growth and improving the crop yield. In general, conventional mineral fertilizers are soluble salts, which easily dissolve in the soil media for plant uptake. However, a large portion of these soluble salts leach to the water resources, resulting in eutrophication and nutrient loss in fertilization. Solid forms of insoluble fertilizers also have been applied but the micronutrients are not so free to be easily bioavailable and easily transport to the water resources. Although, when the plants are in need, these solid minerals are less effective in supplying micronutrients in time due to their large size. To solve these problems, application of nanoparticles could be helpful hypothetically in both providing the micronutrients, minimizing the environmental contamination risks of soluble fertilizers and the problem of less bioavailability of solid fertilizers which is more environmentally benign (Singh et al. 2018; Liu et al. 2014). As the dimensions of materials reduce from a large size below $100 \mathrm{~nm}$, significant changes in characteristics can occur mainly due to an increase in relative surface area (per unit mass) and then an enhanced chemical reactivity (Al-Hakkani 2020).

In recent years, a large number of reports have analyzed the influence of various nanostructures on different crops especially on their early developmental stage, seed germination and seedling development. Seed germination, i.e., the emergence of the radicle and primary root's elongation, is considered as the most sensitive stage of a plant life cycle. Priming with nanoparticle (nano-priming) could lead to positive, negative, or no impacts on germination process, depending on NPs type, size, concentration, duration of the exposure, or the growing conditions. Exact outcomes of the seed priming also consider as a technique to control the hydrate content of the seeds, stimulating metabolic activities for germination (Salah et al. 2015; Szollosi et al. 2020). In priming with NPs, first, the NPs have to penetrate the sclereids barrier of seed coat. Several studies revealed that NPs reach to the plant cells by crossing the intercellular spaces, bounding to a carrier protein, through aquaporin, ion channels, bounding to organic materials or endocytosis via making new pores. The NP-plant interaction may result in morphological and physiological changes, depending on characteristics of NPs (Singh et al. 2018; Aslani et al. 2014). It is also confirmed that some metal-based NPs can cross the seed coat and stimulate the embryonic differentiation through inducing the enzymes which interrupt seed dormancy. NPs may translocate to the other plant parts and cause various structural or functional changes in those parts (Alam et al. 2015). Previous reports also suggested different biochemical mechanisms for positive effect of nanomaterials upon NP exposure such as increased water uptake, remodeling of membrane lipids in seeds enhanced sugar metabolism and energy production, and the stimulated antioxidant defense (Szollosi et al. 2020). The impact of NPs on seed germination is related to their capability to enter the embryonic tissues through the seed coat. This capability is mostly related to the structure of seed coat and differs upon each plant species, physical and chemical characteristics of the ambience (Ko et al. 2017).

There is also another reason for evaluation of NP-plant interactions. Up to now, the amount of NPs existed in the environment is really lower than their toxic concentration. However, as NPs are widely commercialized, the potential biological impacts of NPs should be carefully assessed. As NPs have the potential to find their pathway into the environment and plants continuously interact with soil, water and air, these NPs may penetrate and translocate into the plant (Yang et al. 2017; Rastogi et al. 2017). In this regard, there will be a need to study the impact of NPs on plants.

Metals generally affect seed germination process, biochemical and physiological profiles and plant growth. Essential metals such as Zn, Mg, Cu and Fe are crucial for living cells and their deficiency could lead to damages in cell wall and DNA. Nevertheless, the excessive amounts of these metals or presence of non-essential metals (e.g., Ag) could be toxic due to causing oxidative stress, stimulating loss of membrane integrity, and injuring to proteins and DNA in a phytotoxic manner (Rai et al. 2017).

The objective of this study encompasses assessment potential impact of seven metal-based nanoparticles, which we have biosynthesized, characterized and applied in our previous works (Bayat et al. 2021a; Bayat et al. 2021b; Bayat et al. 2019), on seedling and seedling growth of two popular plants of wheat and flax though a laboratory study. Calcinated zinc oxide (C-ZnO), non-calcinated zinc oxide (NC-ZnO), zinc ( $\mathrm{Zn}$ ), magnesium oxide (MgO), copper (Cu) and iron $(\mathrm{Fe})$ include essential metals which are vital for plant growth. Ag NP is selected due to its extensive use in industry and it could reach to the plants by surface water. Essential and nonessential elements might be absorbed by plant and according to their concentration, may result in toxicity. The effect of NPs also studied in some earlier reports. These nanoparticles showed both promoting and inhibition effects on plants growth (Vanninia et al. 2014; Asanova et al. 2019; Gorczyca et al. 2018). Moreover, the influence of metal salts, which are used as the precursors of the NPs during their biosynthesis, is compared with their counterpart NPs at the same concentrations. To the best of our knowledge, this report is the first to compare the positive and negative effects of these NPs on seedling parameters.

\section{Materials And Methods}


The plant extraction method, synthesis and characterization of applied NPs are described in our previous works (Bayat et al. 2021a; Bayat et al. 2019). In summary, dried strawberry leaves boiled in distilled water, filtered and mixed with NPs precursors. NPs generated by reduction of $0.01 \mathrm{M}$ precursor salt's solutions under heating, continuous stirring and addition of the extract drop by drop. Produced NPs washed with distilled water after centrifuge and dried at room temperature. C-ZnO and $\mathrm{MgO}$ NPs calcinated in furnace at $500^{\circ} \mathrm{C}$ for $4 \mathrm{~h}$. The biosynthesized NPs specified using different characterization techniques including UV-Vis Spectroscopy, XRD, FESEM, EDS, Photon Cross-Correlation Spectroscopy (PCCS) and FT-IR. Biosynthesized NPs, their counterpart precursors, their average sizes and shapes are listed in Table1.

TABLE 1: list of the applied biogenic NPs, their counterpart precursors, the average size and shape of the NPs

\begin{tabular}{|llllllll|}
\hline NP & $\mathrm{C}-\mathrm{ZnO}$ & $\mathrm{NC}-\mathrm{ZnO}$ & $\mathrm{Zn}$ & $\mathrm{MgO}$ & $\mathrm{Ag}$ & $\mathrm{Cu}$ & $\mathrm{Fe}$ \\
\hline Precursor & $\mathrm{Zn}(\mathrm{CH} 2 \mathrm{COO})_{2}$ & $\mathrm{Zn}(\mathrm{CH} 2 \mathrm{COO})_{2}$ & $\mathrm{Zn}(\mathrm{CH} 2 \mathrm{COO})_{2}$ & $\mathrm{MgSO}_{4}$ & $\mathrm{AgNO}_{3}$ & $\mathrm{CuSO}_{4}$ & $\mathrm{FeCl}_{3}$ \\
\hline Ave NP size & 40 & 25 & 100 & 65 & 50 & $180 \times 30$ & $130 \times 20$ \\
Shape & Spherical & spherical & Small sheets & Semi-spherical & spherical & sheets & Small sheets \\
\hline
\end{tabular}

\section{Preparation of priming solutions}

Different concentrations $\left(50,100,150\right.$ ppm) of $\mathrm{NPs}\left(\mathrm{C}-\mathrm{ZnO}, \mathrm{NC}-\mathrm{ZnO}, \mathrm{Zn}, \mathrm{MgO}, \mathrm{AgNO}_{3}, \mathrm{Cu}\right.$ and Fe) and also their counterpart metal salts $\left(\mathrm{Zn}(\mathrm{CH} 2 \mathrm{COO})_{2}\right.$, $\mathrm{MgSO}_{4}, \mathrm{AgNO}_{3}, \mathrm{CuSO}_{4}$ and $\mathrm{FeCl}_{3}$ ) prepared in distilled water and the NPs dispersed by ultrasonic vibrations for 20 minutes. All dilutions were freshly prepared before use.

\section{Preparation of seeds}

Seeds of wheat (Triticum aestivum L.) variety Firuza 40 and flax (Linum usitatissimum) variety Semi Lini were used in this experiment. Each treatment consisted 30 randomly selected seeds with three replications. Seeds were kept in dry place at room temperature prior to use.

(a) Preparation of wheat seeds: Viability of seeds checked visually and then by suspending in distilled water, discarding the seeds floating above were and selecting seeds settled at bottom of the water for further experiment. Seeds immersed in a $5 \%$ sodium hypochlorite solution and rinsed with distilled water after 10 minutes, for surface sterility of the seeds (USEPA 1996). Then seeds soaked in a prepared NPs suspensions or metal salt solution for 12 hours. A set of seeds was soaked in distilled water without providing any treatment as control.

(b) Preparation of flax seeds: Seeds were checked visually for removing damaged seeds from the samples. Then seeds soaked in NPs suspensions or metal salt solutions for 12 hours. A set of seeds was used without providing any treatment as a control and soaked in distilled water.

\section{In vitro germination of seeds}

One piece of filter paper was placed into a Petri plate (10 cm in diameter), and for wetting the paper, $5 \mathrm{ml}$ distilled water was added using a Pasteur pipette. Then 30 nano-primed seeds were transferred onto each filter paper. The Petri plates incubated at room temperature for seven days.

\section{Measurement of physiological indexes}

Germination percentages, shoot length, root length, seedlings length, root-shoot ratio, seedling vigor index (SVI), shoot length stress tolerance index (SLSI) and root length stress tolerance index (RLSI) were calculated at $2^{\text {nd }}$ and $7^{\text {th }}$ days. Means and standard deviations were derived from measurements on three replicates for each treatment and controls. A seed was considered germinated after the emergence of radicles or plumules from the seed coat (Ahmed et al., 2019). The length of roots and shoots were measured using a ruler with centimeter and millimeter scale (fig. 1).

(a) Shoot and root length: At $2^{\text {nd }}$ and $7^{\text {th }}$ days of the experiment, 10 seedlings from petri plate randomly selected to measure shoot and root lengths (Rawat et al. 2018).

(b) Seedling length: is considered as the sum of shoot length and root length of a seed (Rawat et al. 2018).

(c) Germination percentage: The germination percentage was calculated based on the total number of germinated seeds at the day of the experiment. Germination percentage calculated using the following equation (Raskar et al. 2013):

Germination percentage $(\%)=($ average number of germinated seeds/total number of seeds $) \times 100$

(d) Root/Shoot Ratio: The root to shoot ratio for each seedling was calculated as follows (Raskar et al. 2013):

Root/Shoot Ratio = average root length/ average shoot length

(e) Seedling Vigor Index (SVI): The seedling vigor index was computed by adopting the method suggested by Abdul-Baki and Anderson (1973) and expressed as an index number (Ushahra et al. 2013):

Seedling Vigor Index (SVI) = [average root length $(\mathrm{cm})+$ average shoot length $(\mathrm{cm})] \times$ average germination percentage

(f) Shoot length stress tolerance index (SLSI) calculated using following equation (Ahmed et al., 2019; Raskar et al., 2013): 
SLSI $(\%)=$ average shoot length of treated seedlings/average shoot length of control seedlings $\times 100$

(g) Root length stress tolerance index (RLSI) calculated as follow (Ahmed et al. 2019; Raskar et al. 2013):

RLSI $(\%)=$ average root length of treated seedlings/average root length of control seedlings $\times 100$

\section{Statistical analysis}

The obtained data statistically analyzed using Microsoft Excel software (version 2019), SAS and MSTAT-C statistical programs. One-way analysis of variance (ANOVA) applied for performing statistical analysis and p-value $<0.05$ considered as significant. Mean comparison performed by Least Significant Different (LSD) test. Means and standard deviations obtained from measurements on three replicates for control and each treatment.

\section{Results}

In the present research, we carried out experiments to compare the effect of our synthesized biogenic $\mathrm{NPs}(\mathrm{C}-\mathrm{ZnO}, \mathrm{NC}-\mathrm{ZnO}, \mathrm{Zn}, \mathrm{MgO}, \mathrm{Ag}, \mathrm{Cu}$ and Fe) and their counterpart metallic salts $\left(\mathrm{Zn}\left(\mathrm{CH}_{3} \mathrm{COO}\right)_{2}, \mathrm{MgSO}_{4}, \mathrm{AgNO}_{3}, \mathrm{CuSO}_{4}\right.$ and $\left.\mathrm{FeCl}_{3}\right)$ on germination and seedling growth of wheat and flax by considering their effect on different parameters including germination percentage, shoot length, root length, seedlings length, root/shoot ratio, seedling vigor index (SVI), shoot length stress tolerance index (SLSI) and root length stress tolerance index (RLSI). Three different concentrations of 50, 100 and 150ppm were used for seed priming. These concentrations were selected considering previous reports (Gorczyca et al. 2018; Younes et al. 2020). Wheat and flax plantlets were grown In vitro and all the observations were recorded up to 7 days. Results are tabulated in Tables 2 to 9 . We observed that plant growth parameters of wheat and flax, varied considerably among the plants and also different priming solutions with various concentrations. Moreover, both effects of "stimulation" and "phytotoxicity" and in some cases no significant effect of NPs and their counterpart salts was observed on germination and seedling growth. The $\mathrm{p}$-values less than 0.05 indicate there is a significant difference within the results.

\section{Effect of biogenic NPs and their counterpart salts on physiological characteristics of wheat seedling}

Tables 2-5 summarize the effect of priming with biogenic NPs and their counterpart metal salts (precursors) on seed germination parameters of wheat on $2^{\text {nd }}$ and $7^{\text {th }}$ day.

\section{Germination percentage}

The data showed that exposure to different concentrations of biogenic NPs resulted in an increase in germination percentage (G\%) of all the treatments over the control at $2^{\text {nd }}$ day (Table 2). The maximum G\% is related to the seeds primed with Zn NP at concentration of 150 and then $100 p p m$ i.e., 98 and $96 \%$ respectively. Vise a versa, a decrease in $\mathrm{G} \%$ occurred in samples treated with metal salts, comparing to the control, except the sample primed with $50 \mathrm{ppm}$ of zinc acetate in which $\mathrm{G} \%$ was similar to the control (Table 4).

At $7^{\text {th }}$ day, an increase was observed in most of the samples and the highest G\% was related to the concentrations of 50 and $150 \mathrm{ppm}$ of $\mathrm{Zn}$ NPs and also $50 \mathrm{ppm}$ of Cu NPs treated seeds (Table 3). Interestingly, for the samples treated with metal salts, the G\% values are similar or close to the control except to the seeds primed with iron chloride, which showed a concentration dependent increase (Table 5). Among all of the applied NPs, Zn NPs found to be more effective in developing the seed germination of wheat seeds.

\section{Shoot length and SLSI}

At $2^{\text {nd }}$ day of the experiment, the best results of shoot elongation were related to the seeds treated with NC-ZnO NPs among all of the used NPs (Table 2) and also the maximum SLSI caused by 50 and 100ppm of NC-ZnO and 50ppm of Fe NPs treatments (about 115 to $120 \%$ ). In the case of priming with salts (Table 4), the shoot elongations and SLSI values were less than the control, except 100ppm zinc acetate which was close to the control. Ag NPs induced a significant decrease in shoot length and SLSI, both in $2^{\text {nd }}$ and $7^{\text {th }}$ day. According to the Tables 3 and 5 , the maximum shoot lengths and SLSI at $7^{\text {th }}$ day are related to the concentrations of $50 \mathrm{ppm}$ of $\mathrm{Zn}$ and $\mathrm{Fe}$, and also 50 and $150 \mathrm{ppm}$ of $\mathrm{Cu} \mathrm{NPs}$ (108 to $112 \%$ ). In salt primed samples, just priming with $100 \mathrm{ppm}$ of $\mathrm{FeCl} 3 \mathrm{~h}_{3}$ had a

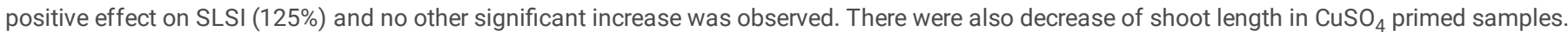

\section{Root length and RLSI}

According to the Tables $2 \& 4$, at the $2^{\text {nd }}$ day of the test, the most significant promoting effect on root length and RLSI is related to the soaking with NC-ZnO NPs. Root lengths of the Zn NPs treated seeds were similar to the control and the other NP treatments had an inverse effect on root elongation. Considering the results of the priming with salts, the increase in root lengths and $\mathrm{RLSI}$ observed in zinc acetate treated seeds, also in $150 \mathrm{ppm}$ of $\mathrm{MgSO}_{4}$ and $\mathrm{AgNO}_{3}$ priming and also in 50ppm of $\mathrm{CuSO}_{4}$ treatments. For seeds treated with $\mathrm{FeCl}_{3}, 50$ ppm $\mathrm{MgSO}_{4}$ and $100 \mathrm{ppm}$ CuSO${ }_{4}$, a significant decline was indicated

In $7^{\text {th }}$ day (Table 3\&5), there were a significant improvement in root length for all of the NP-primed seeds, except Ag NP-primed samples. NC-ZnO had the best effect on root length development and Ag NP showed a dose dependent inhibition effect on root lengths. For metal salt priming cases, the best results in root elongation and RLSI were related to the 100 and $150 \mathrm{ppm}$ of $\mathrm{FeCl}_{3}$ and then 100 and $150 \mathrm{ppm}$ of zinc acetate priming. For the other samples there were $\mathrm{a}$ decrease in root length, mainly in $\mathrm{CuSO}_{4}$ primed seeds.

\section{Seedling length}


In $2^{\text {nd }}$ day (Tables 2\&4), for Ag primed seeds, there were a remarkable decrease in seedling length. Soaking with 100\&150ppm NC-ZnO and 50\&150ppm Zn NPs exhibited an increase in seedling development. Additionally, zinc acetate was the most effective salt in improving seedling length and $\mathrm{CuSO}_{4}$ was the most toxic one. For other samples the results were similar to the control or just a little lower than the control.

In $7^{\text {th }}$ day (Tables 3\&5), for all NP treatments we observed seedling length improvement in comparison with the control, except Ag NP treated seeds. The maximum effect obtained with 150ppm C-ZnO, 100ppm NC-ZnO and 150ppm MgO NPs (about 25\% more than the control).

For metal salt primed seeds, application of $\mathrm{CuSO}_{4}, \mathrm{AgNO}_{3}$ and $\mathrm{MgSO}_{4}$ led to a notable inhibition of seedling growth respectively. FeCl 3 treatments, had the best improving effect in a dose dependent manner.

\section{Root/soot ratio (R/S)}

Under specific conditions, higher proportion of roots can help plants to compete more efficiently for water uptake and soil resources, while a higher proportion of shoots can help plants to collect more light energy (Allaby, 2006). In $2^{\text {nd }}$ day (Table 2\&4), all of the samples had the R/S of more than 1 , indicating root lengths longer than shoot lengths and the values were similar to the control or less than that, except the Ag NP treatments. In Ag NP primed seeds, a great improvement in R/S observed mainly at the concentration of 100ppm which was about 1.5 times more than its respective control and the minimum was related to the $50 \mathrm{ppm}$ Fe NP which was 0.3 times lower than the control. For metal salt treatments, $\mathrm{AgNO}_{3}$ showed the results close to the $\mathrm{Ag}$ NP treatments, zinc acetate had the most effect in R/S values and $\mathrm{CuSO}_{4}$ had a dose dependent decrease. Overall, the R/S in salt treated seeds was more than NP treated ones.

In $7^{\text {th }}$ day, R/S ratios of the NP treated seeds are higher than the control, except 150ppm Ag treated one and the maximum was for 150ppm C-ZnO NP treatments (Table 3). For salt priming, the amounts are less than NP treated seeds and just 100\&150ppm zinc acetate and 110\&150ppm FeCl ${ }_{3}$ root to shoot ratios were higher than the control (Table 5).

\section{SVI}

For evaluating the effect of metal NPs or metal salts on seedling growth, the seedling vigor index (SVI) could be used as a phytotoxicity index (Zhao et al. 2016). In $2^{\text {nd }}$ day, Ag NP priming resulted in minimum values of SVI. NC-ZnO and Zn NPs had values higher than the control and in salt treated seeds SVIs were less than NP treated seeds. The minimum amounts are due to the $\mathrm{CuSO}_{4}$ and $\mathrm{FeCl}_{3}$ treatments. In $7^{\text {th }}$ day, for NP treatments, all SVI amounts were considerably more than the control. The maximum SVI observed in 150ppm of C-ZnO, 50\&150ppm of Zn NP and 150ppm of MgO NP primed samples. For salt treatments, $100 \& 150 \mathrm{ppm}$ of zinc acetate and $150 \mathrm{ppm}$ of $\mathrm{FeCl}_{3}$ solutions had the maximums SVIs. Besides, $\mathrm{CuSO}_{4}$ and $\mathrm{AgNO}_{3}$ showed the most inhibition effect.

TABLE 2 effect of biogenic NPs priming on seed germination parameters of wheat on $2^{\text {nd }}$ day, under different nanoparticle concentrations. Data are presented as mean values $\pm S D$ for three independent experiments. The same letters within a column show no significant difference at a $95 \%$ probability level at the $p<$ 0.05 level. 


\begin{tabular}{|c|c|c|c|c|c|c|c|c|c|}
\hline Nanoparticle & $\begin{array}{l}\text { Concentration } \\
(\mathrm{ppm})\end{array}$ & $\begin{array}{l}\text { Germination } \\
\text { percentage } \\
\text { (G\%) }\end{array}$ & $\begin{array}{l}\text { Shoot } \\
\text { length } \\
\text { (cm) }\end{array}$ & $\begin{array}{l}\text { Root length } \\
\text { (cm) }\end{array}$ & $\begin{array}{l}\text { Seedling } \\
\text { length } \\
(\mathrm{cm})\end{array}$ & $\begin{array}{l}\text { Root to } \\
\text { shoot } \\
\text { ratio }\end{array}$ & SVI & SLSI (\%) & RLSI \\
\hline \multirow[t]{4}{*}{ C-ZNO } & 0 (Control) & $83.33 \pm 3.33 \mathrm{e}$ & $2.64 \pm 0.27 \mathrm{~b}$ & $4.26 \pm 0.27 a b$ & $6.90 \pm 0.45 a b$ & $1.62 \pm 0.16 \mathrm{c}$ & $574.97 \pm 37 c$ & $100.00 \pm 0.00 \mathrm{~b}$ & $100 . c$ \\
\hline & 50 & $90.00 \pm 1.61 b c$ & $2.36 \pm 0.28 c$ & $3.12 \pm 0.41 \mathrm{c}$ & $5.48 \pm 0.56 d$ & $1.33 \pm 0.20 \mathrm{~d}$ & $493.20 \pm 51 \mathrm{de}$ & $89.85 \pm 11.60 \mathrm{~cd}$ & 73.67 \\
\hline & 100 & $86.66 \pm 2.52 \mathrm{~cd}$ & $2.30 \pm 0.30 \mathrm{c}$ & $3.08 \pm 0.29 c$ & $5.38 \pm 0.51 \mathrm{de}$ & $1.35 \pm 0.19 d$ & $466.23 \pm 44 e$ & $87.10 \pm 8.21 d$ & $72.4 c$ \\
\hline & 150 & $93.33 \pm 1.87 b$ & $2.70 \pm 0.18 b$ & $4.34 \pm 0.67 \mathrm{ab}$ & $7.04 \pm 0.77 a b$ & $1.61 \pm 0.23 c$ & $657.04 \pm 71 b$ & $102.76 \pm 7.94 b$ & $102 . c$ \\
\hline \multirow[t]{4}{*}{ NC-ZnO } & 0 (Control) & $83.33 \pm 3.33 \mathrm{e}$ & $2.64 \pm 0.27 b$ & $4.26 \pm 0.27 a b$ & $6.90 \pm 0.45 a b$ & $1.62 \pm 0.16 \mathrm{c}$ & $574.97 \pm 37 c$ & $100.00 \pm 0.00 \mathrm{~b}$ & $100 . c$ \\
\hline & 50 & $93.33 \pm 2.11 \mathrm{~b}$ & $3.18 \pm 0.24 a$ & $4.86 \pm 0.54 a$ & $6.82 \pm 0.75 b$ & $1.53 \pm 0.11 \mathrm{~cd}$ & $750.37 \pm 70 a$ & $120.31 \pm 13.3 b$ & $115 . c$ \\
\hline & 100 & $93.33 \pm 2.37 \mathrm{~b}$ & $3.08 \pm 0.21 \mathrm{a}$ & $4.70 \pm 0.56 a$ & $7.78 \pm 0.72 a$ & $1.52 \pm 0.14 \mathrm{~cd}$ & $726.11 \pm 67 a$ & $117.16 \pm 8.30 \mathrm{a}$ & 110.5 \\
\hline & 150 & $86.66 \pm 1.26 \mathrm{~cd}$ & $2.84 \pm 0.26 a b$ & $4.96 \pm 0.37 a$ & $7.80 \pm 0.56 a$ & $1.75 \pm 0.15 b c$ & $675.94 \pm 48 b$ & $107.57 \pm 9.87 \mathrm{~b}$ & 116.4 \\
\hline \multirow[t]{4}{*}{$\mathrm{Zn}$} & 0 (Control) & $83.33 \pm 3.13 e$ & $2.64 \pm 0.27 b$ & $4.26 \pm 0.11 \mathrm{ab}$ & $6.90 \pm 0.45 a b$ & $1.62 \pm 0.16 \mathrm{c}$ & $574.97 \pm 37 c$ & $100.00 \pm 0.00 \mathrm{~b}$ & $100 . c$ \\
\hline & 50 & $86.66 \pm 3.02 \mathrm{~cd}$ & $2.70 \pm 0.34 b$ & $4.50 \pm 0.79 \mathrm{ab}$ & $7.20 \pm 0.93 a$ & $1.68 \pm 0.34 c$ & $623.95 \pm 80 \mathrm{~b}$ & $102.27 \pm 12.84 b$ & 105.6 \\
\hline & 100 & $96.66 \pm 1.91 \mathrm{ab}$ & $2.50 \pm 0.24 b c$ & $4.42 \pm 0.35 \mathrm{ab}$ & $6.92 \pm 0.19 a b$ & $1.79 \pm 0.32 \mathrm{bc}$ & $668.88 \pm 18 b$ & $94.69 \pm 9.27 \mathrm{c}$ & 103.7 \\
\hline & 150 & $98.88 \pm 2.23 a$ & $2.72 \pm 0.21 b$ & $4.56 \pm 0.38 \mathrm{ab}$ & $7.28 \pm 0.46 a$ & $1.43 \pm 0.19 d$ & $719.84 \pm 46 a$ & $103.03 \pm 8.21 \mathrm{~b}$ & $107 . c$ \\
\hline \multirow[t]{4}{*}{ MgO } & 0 (Control) & $83.23 \pm 3.31 e$ & $2.64 \pm 0.27 b$ & $4.26 \pm 0.12 \mathrm{ab}$ & $6.90 \pm 0.45 a b$ & $1.62 \pm 0.16 \mathrm{c}$ & $574.97 \pm 37 c$ & $100.00 \pm 0.00 \mathrm{~b}$ & $100 . c$ \\
\hline & 50 & $90.00 \pm 1.93 b c$ & $2.44 \pm 0.39 b c$ & $3.84 \pm 0.35 b$ & $6.28 \pm 0.71 b c$ & $1.59 \pm 0.16 c$ & $565.20 \pm 64 \mathrm{~cd}$ & $92.42 \pm 14.81 \mathrm{c}$ & 90.14 \\
\hline & 100 & $93.33 \pm 4.05 b$ & $2.46 \pm 0.30 \mathrm{bc}$ & $3.46 \pm 0.23 b c$ & $5.92 \pm 0.32 c$ & $1.43 \pm 0.23 d$ & $552.51 \pm 30 \mathrm{~cd}$ & $93.18 \pm 11.55 c$ & 81.22 \\
\hline & 150 & $93.31 \pm 3.21 \mathrm{~b}$ & $2.66 \pm 0.27 b$ & $3.82 \pm 0.69 \mathrm{~b}$ & $6.48 \pm 0.93 b c$ & $1.43 \pm 0.16 d$ & $604.78 \pm 87 \mathrm{bc}$ & $100.75 \pm 10.23 b$ & 89.67 \\
\hline \multirow[t]{4}{*}{$\mathrm{Ag}$} & 0 (Control) & $85.00 \pm 2.90 \mathrm{~d}$ & $2.16 \pm 0.28 \mathrm{~cd}$ & $3.83 \pm 0.28 b$ & $6.00 \pm 0.50 c$ & $1.78 \pm 0.20 \mathrm{bc}$ & $510.00 \pm 42 d$ & $100.00 \pm 0.00 \mathrm{~b}$ & $100 . c$ \\
\hline & 50 & $87.00 \pm 1.07 \mathrm{~cd}$ & $1.60 \pm 0.41 \mathrm{e}$ & $3.25 \pm 0.28 b c$ & $4.85 \pm 0.46 f$ & $2.14 \pm 0.61 a b$ & $421.95 \pm 40 \mathrm{e}$ & $74.07 \pm 18.90 \mathrm{e}$ & 84.85 \\
\hline & 100 & $90.01 \pm 1.93 b c$ & $1.40 \pm 0.36 \mathrm{ef}$ & $3.33 \pm 0.28 b c$ & $4.73 \pm 0.25 f$ & $2.53 \pm 0.88 a$ & $426.00 \pm 22 e$ & $64.81 \pm 16.69 \mathrm{fg}$ & 87.03 \\
\hline & 150 & $91.00 \pm 1.89 b c$ & $1.46 \pm 0.32 \mathrm{ef}$ & $3.23 \pm 0.46 \mathrm{bc}$ & $4.70 \pm 0.78 f$ & $2.23 \pm 0.20 \mathrm{ab}$ & $427.70 \pm 71 e$ & $67.90 \pm 14.88 f$ & 84.42 \\
\hline \multirow[t]{4}{*}{$\mathrm{Cu}$} & 0 (Control) & $85.00 \pm 2.90 \mathrm{~d}$ & $2.16 \pm 0.28 \mathrm{~cd}$ & $3.83 \pm 0.28 b$ & $6.00 \pm 0.50 c$ & $1.78 \pm 0.20 \mathrm{bc}$ & $510.00 \pm 42 d$ & $100.00 \pm 0.00 \mathrm{~b}$ & $100 . c$ \\
\hline & 50 & $92.03 \pm 1.68 b$ & $2.20 \pm 0.10 c$ & $3.50 \pm 0.50 \mathrm{bc}$ & $5.70 \pm 0.60 d$ & $1.58 \pm 0.15 \mathrm{c}$ & $524.40 \pm 55 d$ & $101.85 \pm 4.62 b$ & 91.38 \\
\hline & 100 & $87.06 \pm 2.07 \mathrm{~cd}$ & $2.30 \pm 0.21 c$ & $3.53 \pm 0.50 \mathrm{bc}$ & $5.86 \pm 0.71 \mathrm{~cd}$ & $1.51 \pm 0.08 \mathrm{~cd}$ & $510.40 \pm 61 d$ & $108.02 \pm 9.63 b$ & 92.25 \\
\hline & 150 & $90.01 \pm 1.93 b c$ & $2.00 \pm 0.50 \mathrm{~d}$ & $3.73 \pm 0.92 b$ & $5.73 \pm 1.41 \mathrm{~cd}$ & $1.86 \pm 0.11 \mathrm{bc}$ & $516.00 \pm 127 d$ & $92.59 \pm 23.14 \mathrm{c}$ & 97.47 \\
\hline \multirow[t]{4}{*}{$\mathrm{Fe}$} & 0 (Control) & $85.00 \pm 2.90 \mathrm{~d}$ & $2.16 \pm 0.28 \mathrm{~cd}$ & $3.83 \pm 0.28 b$ & $6.00 \pm 0.50 c$ & $1.78 \pm 0.20 \mathrm{bc}$ & $510.00 \pm 42 d$ & $100.00 \pm 0.00 \mathrm{~b}$ & $100 . c$ \\
\hline & 50 & $90.00 \pm 1.93 b c$ & $2.50 \pm 0.43 \mathrm{bc}$ & $3.10 \pm 0.17 c$ & $5.6 \pm 0.40 \mathrm{~d}$ & $1.26 \pm 0.23 \mathrm{de}$ & $504.00 \pm 36 d$ & $115.74 \pm 20.18 a$ & 80.93 \\
\hline & 100 & $89.00 \pm 3.05 \mathrm{c}$ & $1.87 \pm 0.49 \mathrm{de}$ & $2.92 \pm 1.1 \mathrm{~cd}$ & $4.8 \pm 1.53 f$ & $1.54 \pm 0.39 \mathrm{~cd}$ & $427.20 \pm 136$ & $86.80 \pm 22.79 d$ & 76.37 \\
\hline & 150 & $90.01 \pm 1.93 b c$ & $2.10 \pm 0.26 \mathrm{~cd}$ & $3.40 \pm 0.79 b c$ & $5.5 \pm 1.01 d$ & $1.61 \pm 0.27 c$ & $495.00 \pm 91 \mathrm{de}$ & $97.22 \pm 12.24 \mathrm{c}$ & 88.77 \\
\hline$p$ value & - & 0.0050 & 0.0011 & 0.0002 & 0.0059 & 0.0010 & 0.0009 & 0.0016 & 0.000 \\
\hline
\end{tabular}

TABLE 3 effect of biogenic NPs priming on seed germination parameters of wheat on $7^{\text {th }}$ day, under different nanoparticle concentrations. Data are presented as mean values $\pm S D$ for three independent experiments. The same letters within a column show no significant difference at a $95 \%$ probability level at the $p<$ 0.05 level. 


\begin{tabular}{|c|c|c|c|c|c|c|c|c|c|}
\hline Nanoparticle & $\begin{array}{l}\text { Concentration } \\
(\mathrm{ppm})\end{array}$ & $\begin{array}{l}\text { Germination } \\
\text { percentage } \\
\text { (G\%) }\end{array}$ & $\begin{array}{l}\text { Shoot length } \\
(\mathrm{cm})\end{array}$ & $\begin{array}{l}\text { Root length } \\
\text { (cm) }\end{array}$ & $\begin{array}{l}\text { Seedling } \\
\text { length } \\
\text { (cm) }\end{array}$ & $\begin{array}{l}\text { Root to } \\
\text { shoot } \\
\text { ratio }\end{array}$ & SVI & SLSI (\%) & RLSI (' \\
\hline \multirow[t]{4}{*}{ C-ZNO } & 0 (Control) & $83.33 \pm 3.33 \mathrm{~cd}$ & $11.25 \pm 0.61 b c$ & $9.58 \pm 0.49 f$ & $20.83 \pm 0.9 \mathrm{de}$ & $0.85 \pm 0.03 d$ & $1736 \pm 86 \mathrm{~g}$ & $100.00 \pm 0.00 \mathrm{~b}$ & 100.06 \\
\hline & 50 & $90.00 \pm 1.26 b$ & $11.37 \pm 0.47 b c$ & $11.37 \pm 1.37 d$ & $22.75 \pm 0.9 \mathrm{~cd}$ & $1.00 \pm 0.15 c$ & $2047 \pm 86 d$ & $101.11 \pm 4.25 b$ & $118.6 !$ \\
\hline & 100 & $90.06 \pm 3.03 b$ & $11.12 \pm 0.81 \mathrm{c}$ & $10.47 \pm 0.95 e$ & $22.5 \pm 0.5 \mathrm{~cd}$ & $1.05 \pm 0.16 c$ & $2026 \pm 51 d$ & $97.77 \pm 7.25 b c$ & 120.06 \\
\hline & 150 & $93.33 \pm 2.54 a b$ & $11.25 \pm 0.85 b c$ & $14.25 \pm 0.95 a$ & $25.37 \pm 0.75 a$ & $1.29 \pm 0.17 a$ & $2368 \pm 69 a$ & $98.88 \pm 759 b c$ & 144.76 \\
\hline \multirow[t]{4}{*}{ NC-Zno } & 0 (Control) & $83.33 \pm 3.33 \mathrm{~cd}$ & $11.25 \pm 0.61 b c$ & $9.58 \pm 0.49 f$ & $20.83 \pm 0.9 \mathrm{de}$ & $0.85 \pm 0.03 d$ & $1736 \pm 86 \mathrm{~g}$ & $100.00 \pm 0.00 b$ & 100.06 \\
\hline & 50 & $90.40 \pm 2.76 \mathrm{~b}$ & $11.25 \pm 1.04 \mathrm{bc}$ & $13.37 \pm 1.10 \mathrm{~b}$ & $24.62 \pm 1.7 a b$ & $1.19 \pm 0.11 b$ & $2226 \pm 16 b$ & $100.00 \pm 9.25 b$ & $140.7 \varepsilon$ \\
\hline & 100 & $90.03 \pm 1.73 b$ & $11.87 \pm 0.62 b$ & $13.37 \pm 0.75 b$ & $25.25 \pm 0.64 a$ & $1.13 \pm 0.11 b c$ & $2273 \pm 58 b$ & $105.55 \pm 5.59 \mathrm{ab}$ & 139.5; \\
\hline & 150 & $86.66 \pm 2.98 \mathrm{c}$ & $11.60 \pm 0.41 \mathrm{~b}$ & $14.00 \pm 1.83 a$ & $24.60 \pm 1.7 a b$ & $1.20 \pm 0.17 b$ & $2218 \pm 15 b$ & $103.11 \pm 3.71 \mathrm{ab}$ & 144.05 \\
\hline \multirow[t]{4}{*}{ Zn } & 0 (Control) & $83.33 \pm 3.33 \mathrm{~cd}$ & $11.25 \pm 0.61 b c$ & $9.58 \pm 0.49 f$ & $20.83 \pm 0.9 \mathrm{de}$ & $0.85 \pm 0.03 d$ & $1736 \pm 86 \mathrm{~g}$ & $100.00 \pm 0.00 b$ & 100.06 \\
\hline & 50 & $96.66 \pm 2.69 a$ & $12.25 \pm 2.72 a$ & $12.37 \pm 0.47 c$ & $24.62 \pm 2.6 \mathrm{ab}$ & $1.04 \pm 0.21 \mathrm{c}$ & $2380 \pm 25 a$ & $108.88 \pm 24.20 \mathrm{a}$ & 129.1: \\
\hline & 100 & $86.66 \pm 3.25 \mathrm{c}$ & $11.37 \pm 1.10 \mathrm{bc}$ & $12.62 \pm 0.47 \mathrm{c}$ & $24.00 \pm 1.22 b$ & $1.11 \pm 0.12 b c$ & $2079 \pm 10 \mathrm{~cd}$ & $101.11 \pm 9.85 b$ & 131.74 \\
\hline & 150 & $96.66 \pm 1.59 a$ & $11.75 \pm 0.64 b$ & $12.75 \pm 1.0 \mathrm{bc}$ & $24.50 \pm 1.6 \mathrm{ab}$ & $1.08 \pm 0.04 c$ & $2368 \pm 15 a$ & $104.44 \pm 5.73 a b$ & 133.04 \\
\hline \multirow[t]{4}{*}{ MgO } & 0 (Control) & $83.33 \pm 3.33 \mathrm{~cd}$ & $11.25 \pm 0.61 b c$ & $9.58 \pm 0.49 f$ & $20.83 \pm 0.9 \mathrm{de}$ & $0.85 \pm 0.03 d$ & $1736 \pm 86 \mathrm{~g}$ & $100.00 \pm 0.00 b$ & 100.06 \\
\hline & 50 & $93.33 \pm 1.87 a b$ & $11.47 \pm 0.05 b c$ & $12.12 \pm 0.85 c$ & $23.60 \pm 0.8 b c$ & $1.05 \pm 0.07 c$ & $2202 \pm 80 b$ & $102.00 \pm 0.44 b$ & $126.5:$ \\
\hline & 100 & $93.33 \pm 3.23 \mathrm{ab}$ & $10.87 \pm 0.47 d$ & $12.25 \pm 0.50 \mathrm{c}$ & $23.12 \pm 0.47 c$ & $1.12 \pm 0.08 b c$ & $2158 \pm 44 b c$ & $96.66 \pm 4.25 b c$ & $127.8:$ \\
\hline & 150 & $93.33 \pm 2.36 \mathrm{ab}$ & $11.5 \pm 0.57 \mathrm{c}$ & $13.37 \pm 1.37 b$ & $24.87 \pm 1.93 a$ & $1.16 \pm 0.06 b$ & $2321 \pm 18 a$ & $102.22 \pm 5.13 b$ & 139.5 \\
\hline \multirow[t]{4}{*}{$\mathrm{Ag}$} & 0 (Control) & $89.01 \pm 2.56 \mathrm{bc}$ & $11.24 \pm 1.07 b c$ & $8.34 \pm 2.62 \mathrm{~g}$ & $19.58 \pm 3.18 \mathrm{e}$ & $0.74 \pm 0.21 \mathrm{e}$ & $1743 \pm 28 g$ & $100.00 \pm 0.00 b$ & 100.06 \\
\hline & 50 & $89.5 \pm 2.98 b c$ & $10.82 \pm 0.91 d$ & $9.82 \pm 1.57$ ef & $20.65 \pm 2.1 \mathrm{de}$ & $0.90 \pm 0.13 d$ & $1848 \pm 18 f$ & $100.07 \pm 8.47 b$ & 100.06 \\
\hline & 100 & $91.06 \pm 3.02 b$ & $9.92 \pm 0.90 \mathrm{e}$ & $7.84 \pm 1.13 \mathrm{gh}$ & $17.76 \pm 1.79 f$ & $0.79 \pm 0.09 d$ & $1617 \pm 16 \mathrm{~h}$ & $91.69 \pm 8.39 c$ & 79.85 \\
\hline & 150 & $92.33 \pm 3.11 \mathrm{ab}$ & $9.36 \pm 0.84 f$ & $6.20 \pm 1.03 i$ & $15.57 \pm 1.61 \mathrm{~g}$ & $0.66 \pm 0.10$ ef & $1438 \pm 149 i$ & $86.56 \pm 7.85 \mathrm{~cd}$ & 63.211 \\
\hline \multirow[t]{4}{*}{$\mathrm{Cu}$} & 0 (Control) & $89.01 \pm 2.56 b c$ & $11.24 \pm 1.07 b c$ & $8.34 \pm 2.62 \mathrm{~g}$ & $19.58 \pm 3.18 \mathrm{e}$ & $0.74 \pm 0.21 \mathrm{e}$ & $1743 \pm 283 g$ & $100.00 \pm 0.00 \mathrm{~b}$ & 100.06 \\
\hline & 50 & $95.34 \pm 2.44 a$ & $12.17 \pm 0.78 a$ & $10.89 \pm 1.1 \mathrm{de}$ & $23.06 \pm 1.30 \mathrm{c}$ & $0.89 \pm 0.11 d$ & $2052 \pm 11 d$ & $112.49 \pm 7.23 a$ & 110.91 \\
\hline & 100 & $89.66 \pm 2.81 \mathrm{bc}$ & $11.39 \pm 1.05 b c$ & $10.65 \pm 1.54 e$ & $22.04 \pm 2.04 d$ & $0.94 \pm 0.14 d$ & $1976 \pm 18$ de & $105.30 \pm 9.74 a b$ & 108.4 \\
\hline & 150 & $91.1 \pm 1.20 \mathrm{~b}$ & $11.93 \pm 0.90 \mathrm{ab}$ & $12.43 \pm 1.13 b$ & $24.36 \pm 1.7 a b$ & $1.04 \pm 0.09 c$ & $2219 \pm 15 b$ & $110.32 \pm 8.37 a$ & 126.5 \\
\hline \multirow[t]{4}{*}{$\mathrm{Fe}$} & 0 (Control) & $89.01 \pm 2.56$ & $11.24 \pm 1.07 b c$ & $8.34 \pm 2.62 \mathrm{~g}$ & $19.58 \pm 3.18 \mathrm{e}$ & $0.74 \pm 0.21 e$ & $1743 \pm 28 g$ & $100.00 \pm 0.00 \mathrm{~b}$ & 100.06 \\
\hline & 50 & $91.3 \pm 1.95 b$ & $11.98 \pm 0.58 \mathrm{ab}$ & $11.14 \pm 0.78 d$ & $23.13 \pm 1.15 c$ & $0.93 \pm 0.06 \mathrm{~cd}$ & $2112 \pm 10 c$ & $110.78 \pm 5.43 a$ & $113.4 !$ \\
\hline & 100 & $91.85 \pm 3.03 b$ & $10.96 \pm 0.80 \mathrm{~cd}$ & $10.67 \pm 1.1 \mathrm{de}$ & $21.64 \pm 1.58 d$ & $0.97 \pm 0.10 \mathrm{~cd}$ & $1987 \pm 14$ de & $101.33 \pm 7.43 b$ & 108.7: \\
\hline & 150 & $91.23 \pm 1.23 b$ & $11.32 \pm 1.40 \mathrm{bc}$ & $10.63 \pm 1.0 \mathrm{de}$ & $21.95 \pm 1.58 d$ & $0.95 \pm 0.15 \mathrm{~cd}$ & $2002 \pm 144 d$ & $104.62 \pm 12.9 \mathrm{ab}$ & 108.2; \\
\hline$P$ value & & 0.0100 & 0.0008 & 0.0066 & 0.0003 & 0.0040 & 0.0009 & 0.0160 & $0.003 \varepsilon$ \\
\hline
\end{tabular}

TABLE 4 Effect of metal salt (precursors) priming on seed germination parameters of wheat on $2^{\text {nd }}$ day, under different nanoparticle concentrations. Data are presented as mean values \pm SD for three independent experiments. The same letters within a column show no significant difference at a $95 \%$ probability level at the $p<0.05$ level. 


\begin{tabular}{|c|c|c|c|c|c|c|c|c|c|}
\hline Metal salt & $\begin{array}{l}\text { Concentration } \\
\text { (ppm) }\end{array}$ & $\begin{array}{l}\text { Germination } \\
\text { percentage } \\
\text { (G\%) }\end{array}$ & $\begin{array}{l}\text { Shoot } \\
\text { length } \\
(\mathrm{cm})\end{array}$ & $\begin{array}{l}\text { Root length } \\
\text { (cm) }\end{array}$ & $\begin{array}{l}\text { Seedling } \\
\text { length } \\
(\mathrm{cm})\end{array}$ & $\begin{array}{l}\text { Root to } \\
\text { shoot } \\
\text { ratio }\end{array}$ & SVI & SLSI (\%) & RLSI (\%) \\
\hline \multirow[t]{4}{*}{$\mathrm{Zn}\left(\mathrm{CH}_{3} \mathrm{CO}_{2}\right)_{2}$} & 0 (Control) & $98.88 \pm 1.92 a$ & $1.78 \pm 0.64 a$ & $2.41 \pm 0.37 \mathrm{~cd}$ & $4.20 \pm 0.69 b c$ & $1.47 \pm 0.40 \mathrm{de}$ & $415 \pm 6 a b$ & $100.00 \pm 0.00 a$ & $100.00 \pm 0$ \\
\hline & 50 & $98.88 \pm 1.92 a$ & $1.7 \pm 0.20 \mathrm{a}$ & $3.00 \pm 0.33 b$ & $4.70 \pm 0.36 b$ & $1.78 \pm 0.30 \mathrm{~cd}$ & $464 \pm 35 a$ & $95.50 \pm 11.23 a b$ & $124.48 \pm 1$ \\
\hline & 100 & $93.33 \pm 2.65 \mathrm{c}$ & $1.78 \pm 0.21 a$ & $3.35 \pm 0.27 a$ & $5.13 \pm 0.38 a$ & $1.89 \pm 0.23 c$ & $467 \pm 36 a$ & $100.18 \pm 12.00 \mathrm{a}$ & $139.00 \pm 1$ \\
\hline & 150 & $96.66 \pm 2.92 b$ & $1.48 \pm 0.09 b$ & $2.81 \pm 0.54 \mathrm{c}$ & $4.26 \pm 0.54 b c$ & $1.88 \pm 0.41 \mathrm{c}$ & $412 \pm 5 a b$ & $83.33 \pm 5.52 \mathrm{c}$ & $115.49 \pm 2$ \\
\hline \multirow[t]{4}{*}{ MgSO4 } & 0 (Control) & $98.88 \pm 1.92 a$ & $1.78 \pm 0.64 a$ & $2.41 \pm 0.37 \mathrm{~cd}$ & $4.20 \pm 0.69 b c$ & $1.47 \pm 0.40 \mathrm{de}$ & $415 \pm 6 a b$ & $100.00 \pm 0.00 a$ & $100.00 \pm 0$ \\
\hline & 50 & $86.66 \pm 3.02 e$ & $1.25 \pm 0.16 d$ & $1.55 \pm 0.10 \mathrm{e}$ & $2.80 \pm 0.22 \mathrm{ef}$ & $1.25 \pm 0.14 f$ & $242 \pm 19 d$ & $70.22 \pm 9.23 d$ & $64.31 \pm 4.8$ \\
\hline & 100 & $93.33 \pm 2.65 \mathrm{c}$ & $1.58 \pm 0.24 a b$ & $2.75 \pm 0.30 \mathrm{c}$ & $4.33 \pm 0.18 b c$ & $1.78 \pm 0.40 \mathrm{~cd}$ & $404 \pm 12 b$ & $88.95 \pm 13.49 b c$ & $114.10 \pm 1$ \\
\hline & 150 & $96.66 \pm 1.92 b$ & $1.51 \pm 0.16 b$ & $3.16 \pm 0.25 \mathrm{ab}$ & $4.68 \pm 0.38 b$ & $2.09 \pm 0.17 b$ & $452 \pm 36 a$ & $85.20 \pm 9.00 b c$ & $131.39 \pm 1$ \\
\hline \multirow[t]{4}{*}{ AgNO3 } & 0 (Control) & $98.88 \pm 1.92 a$ & $1.78 \pm 0.64 a$ & $2.41 \pm 0.37 \mathrm{~cd}$ & $4.20 \pm 0.69 b c$ & $1.47 \pm 0.40 \mathrm{de}$ & $415 \pm 6 a b$ & $100.00 \pm 0.00 a$ & $100.00 \pm 0$ \\
\hline & 50 & $83.33 \pm 3.25 f$ & $1.38 \pm 0.20 b c$ & $2.91 \pm 0.20 b c$ & $4.30 \pm 0.24 b c$ & $2.16 \pm 0.45 a b$ & $358 \pm 20 c$ & $77.71 \pm 11.49 \mathrm{~cd}$ & $121.02 \pm 8$ \\
\hline & 100 & $90.12 \pm 2.98 d$ & $1.08 \pm 0.13 d$ & $2.38 \pm 0.37 \mathrm{~cd}$ & $3.46 \pm 0.34 d$ & $2.23 \pm 0.51 a$ & $312 \pm 31 \mathrm{~cd}$ & $60.86 \pm 7.46 \mathrm{e}$ & $98.89 \pm 15$ \\
\hline & 150 & $96.66 \pm 2.92 b$ & $1.31 \pm 0.21 b c$ & $2.78 \pm 0.34 \mathrm{c}$ & $4.10 \pm 0.4 c c$ & $2.16 \pm 0.41 \mathrm{ab}$ & $396 \pm 40 \mathrm{~b}$ & $73.97 \pm 12.00 \mathrm{~d}$ & $115.49 \pm 1$ \\
\hline \multirow[t]{4}{*}{ CuSO4 } & 0 (Control) & $98.88 \pm 1.92 a$ & $1.78 \pm 0.64 a$ & $2.41 \pm 0.37 \mathrm{~cd}$ & $4.20 \pm 0.69 b c$ & $1.47 \pm 0.40 \mathrm{de}$ & $415 \pm 6 a b$ & $100.00 \pm 0.00 a$ & $100.00 \pm 0$ \\
\hline & 50 & $90.89 \pm 3.12 d$ & $1.53 \pm 0.08 \mathrm{bc}$ & $3.11 \pm 0.37 b$ & $4.65 \pm 0.37 b$ & $2.03 \pm 0.28 b$ & $422 \pm 3 a b$ & $86.14 \pm 4.58 b c$ & $129.32 \pm 1$ \\
\hline & 100 & $90.23 \pm 3.65 d$ & $1.31 \pm 0.29 b c$ & $1.63 \pm 0.48 \mathrm{e}$ & $2.95 \pm 0.66 e$ & $1.26 \pm 0.33 f$ & $266 \pm 60 d$ & $73.97 \pm 16.44 d$ & $67.77 \pm 19$ \\
\hline & 150 & $96.66 \pm 2.92 b$ & $1.20 \pm 0.18 d$ & $1.45 \pm 0.19 f$ & $2.65 \pm 0.18 \mathrm{fg}$ & $1.24 \pm 0.30 f$ & $256 \pm 1 d$ & $67.41 \pm 10.65 \mathrm{de}$ & $60.16 \pm 8.1$ \\
\hline \multirow[t]{4}{*}{$\mathrm{FeCl} 3$} & 0 (Control) & $98.88 \pm 1.92 a$ & $1.78 \pm 0.64 a$ & $2.41 \pm 0.37 \mathrm{~cd}$ & $4.20 \pm 0.69 \mathrm{~cd}$ & $1.47 \pm 0.40 \mathrm{de}$ & $415 \pm 6 \mathrm{~cd}$ & $100.00 \pm 0.00 a$ & $100.00 \pm 0$ \\
\hline & 50 & $90.62 \pm 3.02 \mathrm{~d}$ & $1.61 \pm 0.17 a$ & $1.69 \pm 0.10 \mathrm{e}$ & $3.29 \pm 0.10 \mathrm{~d}$ & $1.25 \pm 0.13 f$ & $299 \pm 9 d$ & $90.90 \pm 6.56 \mathrm{~b}$ & $70.42 \pm 2.8$ \\
\hline & 100 & $94.83 \pm 3.03 \mathrm{c}$ & $1.41 \pm 0.38 b$ & $1.58 \pm 0.50 \mathrm{e}$ & $2.98 \pm 0.64 e$ & $1.61 \pm 0.44 d$ & $283 \pm 57 d$ & $79.54 \pm 14.42 c$ & $76.99 \pm 11$ \\
\hline & 150 & $98.33 \pm 2.65 a$ & $1.44 \pm 0.46 \mathrm{~b}$ & $2.07 \pm 0.49 d$ & $3.52 \pm$ & $1.81 \pm 0.67 \mathrm{c}$ & $346 \pm 49 c$ & $81.06 \pm 17.48 \mathrm{c}$ & $85.91 \pm 11$ \\
\hline$P$ value & & 0.0008 & 0.0111 & 0.0101 & 0.0022 & 0.0009 & 0.0100 & 0.0061 & 0.0004 \\
\hline
\end{tabular}

TABLE 5 Effect of metal salt (precursors) priming on seed germination parameters of wheat on $7^{\text {th }}$ day, under different nanoparticle concentrations. Data are presented as mean values \pm SD for three independent experiments. The same letters within a column show no significant difference at a $95 \%$ probability level at the $p<0.05$ level. 


\begin{tabular}{|c|c|c|c|c|c|c|c|c|c|}
\hline Metal salt & $\begin{array}{l}\text { Concentration } \\
(\mathrm{ppm})\end{array}$ & $\begin{array}{l}\text { Germination } \\
\text { percentage } \\
\text { (G\%) }\end{array}$ & $\begin{array}{l}\text { Shoot } \\
\text { length } \\
(\mathrm{cm})\end{array}$ & $\begin{array}{l}\text { Root length } \\
(\mathrm{cm})\end{array}$ & $\begin{array}{l}\text { Seedling } \\
\text { length } \\
\text { (cm) }\end{array}$ & $\begin{array}{l}\text { Root to } \\
\text { shoot } \\
\text { ratio }\end{array}$ & SVI & SLSI (\%) & RLSI (\%) \\
\hline $\mathrm{Zn}$ & 0 (Control) & $98.88 \pm 1.92 a$ & $10.03 \pm 0.70 b$ & $8.99 \pm 0.84 c$ & $19.00 \pm 1.4 d$ & $0.89 \pm 0.07 b$ & $1881 \pm 141 \mathrm{c}$ & $100.00 \pm 0.0 b$ & $100.00 \pm 0$ \\
\hline \multirow[t]{3}{*}{$\left(\mathrm{CH}_{3} \mathrm{CO}_{2}\right)_{2}$} & 50 & $98.66 \pm 2.10 \mathrm{a}$ & $10.00 \pm 1.47 b$ & $8.26 \pm 1.67 \mathrm{~cd}$ & $18.26 \pm 2.41 \mathrm{e}$ & $0.83 \pm 0.19 c$ & $1802 \pm 237 \mathrm{c}$ & $99.70 \pm 14.71 b$ & $91.95 \pm 18$ \\
\hline & 100 & $97.66 \pm 2.65 a$ & $10.32 \pm 0.82 b$ & $10.60 \pm 1.33 b$ & $20.92 \pm 1.96 b$ & $1.02 \pm 0.10 \mathrm{a}$ & $2043 \pm 192 a$ & $102.90 \pm 8.2 a b$ & $114.81 \pm 9$ \\
\hline & 150 & $98.88 \pm 1.92 a$ & $9.85 \pm 1.18 b c$ & $10.58 \pm 1.63 b$ & $20.43 \pm 2.4 b c$ & $1.07 \pm 0.14 a$ & $2020 \pm 242 a$ & $98.20 \pm 11.82 b$ & $117.72 \pm 1$ \\
\hline \multirow[t]{4}{*}{ MgSO4 } & 0 (Control) & $98.88 \pm 1.92 a$ & $10.03 \pm 0.70 b$ & $8.99 \pm 0.84 c$ & $19.03 \pm 1.43 d$ & $0.89 \pm 0.09 b$ & $1881 \pm 112 c$ & $100.00 \pm 0.0 b$ & $100.00 \pm 0$ \\
\hline & 50 & $95.02 \pm 3.02 b c$ & $8.62 \pm 2.59 \mathrm{~cd}$ & $7.45 \pm 1.80 \mathrm{~d}$ & $16.07 \pm 4.30 f$ & $0.90 \pm 0.17 b$ & $1526 \pm 409 e$ & $85.95 \pm 25.8 \mathrm{~cd}$ & $82.86 \pm 20$ \\
\hline & 100 & $98.33 \pm 2.25 a$ & $10.07 \pm 0.56 \mathrm{~b}$ & $8.73 \pm 1.45 \mathrm{~cd}$ & $18.80 \pm 1.5 \mathrm{de}$ & $0.86 \pm 0.15 b c$ & $1843 \pm 155 c$ & $100.46 \pm 5.67 \mathrm{~b}$ & $98.09 \pm 16$ \\
\hline & 150 & $98.88 \pm 1.92 a$ & $9.73 \pm 1.08 \mathrm{c}$ & $8.48 \pm 1.92 \mathrm{~cd}$ & $18.21 \pm 2.86 e$ & $0.86 \pm 0.13 b c$ & $1639 \pm 257 d$ & $97.01 \pm 10.8 \mathrm{bc}$ & $94.37 \pm 21$ \\
\hline \multirow[t]{4}{*}{ AgNO3 } & 0 (Control) & $98.88 \pm 1.92 a$ & $10.03 \pm 0.70 b$ & $8.99 \pm 0.84 c$ & $19.01 \pm 1.41 d$ & $0.89 \pm 0.05 b$ & $1881 \pm 121 \mathrm{c}$ & $100.00 \pm 0.0 b$ & $100.00 \pm 0$ \\
\hline & 50 & $97.06 \pm 3.25 \mathrm{ab}$ & $9.28 \pm 0.72 c$ & $7.10 \pm 1.45 \mathrm{~d}$ & $16.39 \pm 2.05 f$ & $0.76 \pm 0.11 d$ & $1591 \pm 199 \mathrm{de}$ & $92.57 \pm 7.24 \mathrm{c}$ & $79.05 \pm 16$ \\
\hline & 100 & $97.03 \pm 2.98 \mathrm{ab}$ & $7.70 \pm 1.07 d$ & $4.62 \pm 0.90 \mathrm{e}$ & $12.33 \pm 1.45 \mathrm{~g}$ & $0.61 \pm 0.15 e$ & $1196 \pm 140$ ef & $76.85 \pm 10.72 d$ & $51.44 \pm 10$ \\
\hline & 150 & $98.88 \pm 1.92 a$ & $8.47 \pm 1.04 \mathrm{~cd}$ & $4.25 \pm 1.13 e$ & $12.72 \pm 1.88 \mathrm{~g}$ & $0.50 \pm 0.11 f$ & $1258 \pm 186 e$ & $84.49 \pm 10.4 \mathrm{~cd}$ & $47.27 \pm 12$ \\
\hline \multirow[t]{4}{*}{ CuSO4 } & 0 (Control) & $98.88 \pm 1.92 a$ & $10.03 \pm 0.70 b$ & $8.99 \pm 0.84 c$ & $19.02 \pm 1.43 d$ & $0.89 \pm 0.04 b$ & $1881 \pm 141 \mathrm{c}$ & $100.00 \pm 0.0 b$ & $100.00 \pm 0$ \\
\hline & 50 & $97.06 \pm 3.12 \mathrm{ab}$ & $7.73 \pm 2.77 d$ & $4.53 \pm 2.17 \mathrm{e}$ & $12.27 \pm 4.7 \mathrm{~g}$ & $0.58 \pm 0.17 e$ & $1191 \pm 457$ ef & $77.15 \pm 27.67 d$ & $50.48 \pm 24$ \\
\hline & 100 & $96.66 \pm 2.92 b$ & $7.83 \pm 1.29 \mathrm{~d}$ & $1.87 \pm 0.74 f$ & $9.70 \pm 1.88 \mathrm{~h}$ & $0.23 \pm 0.07 \mathrm{~g}$ & $938 \pm 182 \mathrm{~g}$ & $78.09 \pm 12.87 d$ & $20.83 \pm 8.3$ \\
\hline & 150 & $98.88 \pm 1.92 a$ & $5.55 \pm 0.82 e$ & $1.22 \pm 0.65 f$ & $6.77 \pm 0.87 i$ & $0.23 \pm 0.14 \mathrm{~g}$ & $670 \pm 86 \mathrm{~h}$ & $55.33 \pm 8.27 e$ & $13.66 \pm 7.3$ \\
\hline \multirow[t]{4}{*}{$\mathrm{FeCl} 3$} & 0 (Control) & $98.88 \pm 1.92 a$ & $10.03 \pm 0.70 b$ & $8.99 \pm 0.84 c$ & $19.02 \pm 1.43 d$ & $0.89 \pm 0.04 b$ & $1881 \pm 141 \mathrm{c}$ & $100.00 \pm 0.0 b$ & $100.00 \pm 0$ \\
\hline & 50 & $86.66 \pm 2.96 d$ & $11.12 \pm 0.25 a$ & $8.25 \pm 2.17 \mathrm{~cd}$ & $19.37 \pm 1.93 d$ & $0.74 \pm 0.20 \mathrm{~d}$ & $1679 \pm 167 d$ & $98.88 \pm 2.22 b$ & $86.11 \pm 22$ \\
\hline & 100 & $90.00 \pm 3.03 \mathrm{~cd}$ & $11.15 \pm 0.30 \mathrm{a}$ & $10.75 \pm 1.84 b$ & $21.90 \pm 1.79 b$ & $0.96 \pm 0.17 a b$ & $1971 \pm 16 a b$ & $125.43 \pm 3.37 a$ & $112.21 \pm 1$ \\
\hline & 150 & $93.33 \pm 2.65 \mathrm{c}$ & $11.12 \pm 0.47 a$ & $11.25 \pm 0.64 a$ & $22.37 \pm 0.62 a$ & $1.01 \pm 0.08 a$ & $2088 \pm 58 a$ & $98.88 \pm 4.15 b$ & $117.43 \pm 6$ \\
\hline$P$ value & & 0.0200 & 0.0000 & 0.0055 & 0.0007 & 0.0108 & 0.0077 & 0.0100 & 0.0006 \\
\hline
\end{tabular}

Effect of biogenic NPs and their counterpart salts on physiological characteristics of flax seedling

Tables 6-9 summarize the effect of priming with biogenic NPs and their counterpart metal salts (precursors) on seed germination parameters of flax on $2^{\text {nd }}$ and $7^{\text {th }}$ day.

Germination percentage

Considering the results of the Tables $6 \& 8$, in early stages of seedling, the maximum seed germination percentage (90\%) was related to the flax seeds soaked with 100ppm C-ZnO NPs and all of the other treatments showed G\% similar or less than the control. The minimum G\% was related to the seeds soaked with 100 ppm of Cu NPs. Among metal salt, zinc acetate had improving effect on $\mathrm{G} \%$ and except $100 \mathrm{ppm}$ of $\mathrm{CuSO}_{4}$ and $\mathrm{FeCl}_{3} \mathrm{primed}$ seeds, no increase in $\mathrm{G} \%$ was observed with respect to the control. At $7^{\text {th }}$ day of the experiment (Tables 7\&9), just 150ppm of C-ZnO NPs suspension had improving effect over the control and all of the other NP treatments showed inhibition effect. Among salts, $\mathrm{G} \%$ of the $150 \mathrm{ppm}$ of $\mathrm{MgSO}_{4}$ treated seeds was close to the control and the others were less than the control. 150ppm of $\mathrm{AgNO}_{3}$ had such a severe toxic effect so no gemination was observed and there are not any data reported in following for this case.

\section{Shoot length and SLSI}

According to the Tables $6 \& 8$, at $2^{\text {nd }}$ day of the test, all of the NP treatments showed enhancement in shoot length over the control. The most effective NP was $\mathrm{NC}-\mathrm{ZnO}$ and the less effective one was $\mathrm{Ag}$. The most effective salts in improving shoot length were $\mathrm{MgSO}_{4}$ and then zinc acetate. 50ppm of $\mathrm{AgNO}_{3}$ was also very effective in this regard and 100ppm concentration of $\mathrm{AgNO}_{3}$ and $\mathrm{CuSO}_{4}$ had the worst effect on shoot elongation. Tables $7 \& 9$ record the results of the $7^{\text {th }}$ day of the experiment which show that the most effective NP in shoot length development of flax seeds was Ag.

$\mathrm{MgSO}_{4}$ and $\mathrm{FeCl}_{3}$ were the most effective in shoot length development and $100 \mathrm{ppm} \mathrm{AgNO}_{3}$ was the most toxic one, completely inhibiting the germination of seeds.

\section{Root length and RLSI}

Flax seeds responded differently toward the treatment at various concentrations of NPs. At $2^{\text {nd }}$ day (Table $6 \& 8$ ), Zn NPs had the best effect on root growth. Ag and Fe NPs had the most inhibition effect. $\mathrm{MgSO}_{4}$ was the most effective salt in root length development and $\mathrm{CuSO}_{4}$ was the most toxic one. At $7^{\text {th }}$ day of the 
experiment, a significant increase in root length observed in most of the treatments, especially in Zn NPs treated samples and the root lengths were about 2 to 3 times higher than the control which is considered as a great positive effect. Cu 100ppm besides 50 and 150ppm concentrations of Fe NPs showed a notable toxic effect on root length parameter. Zinc acetate and $\mathrm{MgSO}_{4}$ solutions induced a significant root length increase of about twofold over the control. 100 and $150 \mathrm{ppm}$ of $\mathrm{CuSO}_{4}$ priming inhibited the root growth to the lengths about $27 \%$ of the control.

\section{Seedling length}

Like root and shoot length, the best results of seedling length is related to Zn NPs treated samples. After 48h (Tables 6\&8), Ag and Fe had seedling lengths less than the control. For $\mathrm{AgNO}_{3}$ primed samples, 50ppm concentration resulted in maximum seedling length and 100ppm resulted in no seedling. Moreover, $\mathrm{MgSO}_{4}$ showed the best increasing effect in seedling length. After the first week (Tables 7\&9), in the case of nano-primed seeds, except Cu and Fe primed samples which had seedling lengths less than the control, the other treatments had seedling lengths more than the control. Zinc acetate and $\mathrm{MgSO}_{4}$ were most effective in increase of seedling length and the minimum was related to the $100 \mathrm{ppm} \mathrm{AgNO}_{3}$ treatments.

\section{Root/Shoot ratio}

In early stages of flax seedling, the shoot grew with a higher speed in comparison with root. Considering the results of the Tables $6 \& 8$, at $2^{\text {nd }}$ day, all of the R/S values were less than the control in NP and salt primed samples. At $7^{\text {th }}$ day, except Fe NPs and 100-150ppm Cu NPs primed seeds, the other samples had R/S more than the control. 100 and 150ppm of $\mathrm{CuSO}_{4}$ (like $\mathrm{Cu} \mathrm{NP}$ ) and 100ppm of $\mathrm{FeCl}_{3}$ treated seeds had R/S less than the control.

\section{SVI}

At $2^{\text {nd }}$ day of the experiment, maximum SVI was related to the 150ppm C-ZnO and 50ppm of Zn NPs due to their high seedling length values and the minimum was related to the 100ppm of Cu NPs primed samples. At $7^{\text {th }}$ day, Fe NPs primed samples had the minimum SVIs due to their minimum seedling lengths. $\mathrm{MgSO}_{4}$ primed samples had the maximum SVIs and 50ppm zinc acetate and $100 \mathrm{ppm} \mathrm{AgNO}_{3}$ had the minimum SVIs.

TABLE 6 Effect of biogenic NPs priming on seed germination parameters of flax on $2^{\text {nd }}$ day, under different nanoparticle concentrations. Data are presented as mean values $\pm S D$ for three independent experiments. The same letters within a column show no significant difference at a $95 \%$ probability level at the $p<0.05$ level. 


\begin{tabular}{|c|c|c|c|c|c|c|c|c|c|}
\hline Nanoparticle & $\begin{array}{l}\text { Concentration } \\
\text { (ppm) }\end{array}$ & $\begin{array}{l}\text { Germination } \\
\text { percentage } \\
\text { (G\%) }\end{array}$ & $\begin{array}{l}\text { Shoot } \\
\text { length } \\
(\mathrm{cm})\end{array}$ & $\begin{array}{l}\text { Root length } \\
\text { (cm) }\end{array}$ & $\begin{array}{l}\text { Seedling } \\
\text { length } \\
\text { (cm) }\end{array}$ & $\begin{array}{l}\text { Root to } \\
\text { shoot } \\
\text { ratio }\end{array}$ & SVI & SLSI (\%) & RL: \\
\hline Non(Control) & 0 & $86.61 \pm 2.12 b$ & $0.32 \pm 0.04 \mathrm{ef}$ & $2.24 \pm 0.28 \mathrm{~cd}$ & $2.56 \pm 0.28 c$ & $7.10 \pm 1.31 a$ & $221.84 \pm 24.96 c$ & $100.00 \pm 0.00 f$ & 101 \\
\hline \multirow[t]{3}{*}{ C-Zno } & 50 & $86.56 \pm 2.23 b$ & $0.47 \pm 0.04 \mathrm{de}$ & $1.84 \pm 0.47 e$ & $2.32 \pm 0.49 \mathrm{~cd}$ & $3.83 \pm 0.87 d$ & $201.05 \pm 43.06 \mathrm{~cd}$ & $150.00 \pm 13.97 e$ & 82. \\
\hline & 100 & $90.00 \pm 1.93 a$ & $0.66 \pm 0.11 b c$ & $2.52 \pm 0.20 \mathrm{c}$ & $3.18 \pm 0.25 b$ & $3.90 \pm 0.72 d$ & $286.20 \pm 23.29 b$ & $206.25 \pm 35.63 b$ & $11:$ \\
\hline & 150 & $83.33 \pm 3.3 b c$ & $0.74 \pm 0.16 b$ & $3.12 \pm 0.41 \mathrm{a}$ & $3.86 \pm 0.49 a$ & $4.35 \pm 1.02 \mathrm{~cd}$ & $321.65 \pm 41.07 a$ & $231.25 \pm 52.29 a b$ & $13 !$ \\
\hline \multirow[t]{3}{*}{ NC-Zno } & 50 & $80.03 \pm 3.33 c$ & $0.64 \pm 0.18 c$ & $2.74 \pm 0.37 b c$ & $3.38 \pm 0.25 b$ & $4.73 \pm 1.99 \mathrm{c}$ & $270.50 \pm 20.71 b$ & $200.00 \pm 56.78 b$ & $12:$ \\
\hline & 100 & $80.11 \pm 2.98$ & $0.84 \pm 0.15 a$ & $2.32 \pm 0.15 \mathrm{~cd}$ & $3.20 \pm 0.22 b$ & $2.87 \pm 0.50 \mathrm{e}$ & $256.35 \pm 17.91 b c$ & $262.5 \pm 47.39 a$ & $10 !$ \\
\hline & 150 & $83.43 \pm 3.33 c$ & $0.82 \pm 0.10 a$ & $2.20 \pm 0.23 \mathrm{~cd}$ & $3.02 \pm 0.19 b$ & $2.73 \pm 0.55 e$ & $251.65 \pm 16.02 b c$ & $256.25 \pm 34.23 a$ & 98. \\
\hline \multirow[t]{3}{*}{$\mathrm{Zn}$} & 50 & $86.66 \pm 2.12 b$ & $0.74 \pm 0.25 b$ & $3.00 \pm 0.50 \mathrm{ab}$ & $3.74 \pm 0.68 a$ & $4.33 \pm 1.22 b c$ & $324.10 \pm 58.96 a$ & $231.25 \pm 78.43 a b$ & 13: \\
\hline & 100 & $73.43 \pm 4.01 d$ & $0.70 \pm 0.12 b$ & $2.50 \pm 0.60 c$ & $3.20 \pm 0.51 b$ & $3.78 \pm 1.54 d$ & $234.65 \pm 38.10 c$ & $218.75 \pm 38.27 b$ & $11^{\circ}$ \\
\hline & 150 & $73.33 \pm 4.04 d$ & $0.70 \pm 0.21 b$ & $3.08 \pm 0.40 \mathrm{ab}$ & $3.78 \pm 0.59 a$ & $4.61 \pm 0.99 c$ & $277.18 \pm 43.50 b$ & $218.75 \pm 66.29 b$ & 13: \\
\hline \multirow[t]{3}{*}{ MgO } & 50 & $76.82 \pm 3.15 d$ & $0.44 \pm 0.13 \mathrm{de}$ & $2.30 \pm 0.62 \mathrm{~cd}$ & $2.74 \pm 0.71 \mathrm{c}$ & $5.46 \pm 1.74 b$ & $210.04 \pm 54.63 \mathrm{~cd}$ & $137.50 \pm 41.92 \mathrm{de}$ & 10: \\
\hline & 100 & $80.00 \pm 2.45 c$ & $0.56 \pm 0.08 d$ & $2.30 \pm 0.27 \mathrm{~cd}$ & $2.86 \pm 0.25 b c$ & $4.20 \pm 0.88 c$ & $228.80 \pm 20.07 c$ & $175.00 \pm 27.5 c e$ & 10: \\
\hline & 150 & $73.33 \pm 3.25 d$ & $0.66 \pm 0.08 b c$ & $2.42 \pm 0.37 \mathrm{c}$ & $3.08 \pm 0.37 b$ & $3.74 \pm 0.87 d$ & $225.85 \pm 27.14 \mathrm{c}$ & $206.25 \pm 27.95 b$ & 10 \\
\hline \multirow[t]{3}{*}{$\mathrm{Ag}$} & 50 & $83.43 \pm 3.20 c$ & $0.36 \pm 0.15$ ef & $1.63 \pm 0.65 e f$ & $2.00 \pm 0.75 \mathrm{de}$ & $4.65 \pm 1.31 \mathrm{c}$ & $166.66 \pm 62.91 \mathrm{e}$ & $114.58 \pm 47.73 f$ & 72 \\
\hline & 100 & $83.83 \pm 3.35 c$ & $0.44 \pm 0.13 \mathrm{de}$ & $1.66 \pm 0.20 \mathrm{ef}$ & $2.10 \pm 0.1 d$ & $4.52 \pm 3.06 \mathrm{c}$ & $174.99 \pm 8.33 \mathrm{de}$ & $137.50 \pm 41.92$ ef & 74. \\
\hline & 150 & $80.24 \pm 2.86 c$ & $0.46 \pm 0.6 c-e$ & $1.42 \pm 0.35 \mathrm{fg}$ & $1.88 \pm 0.44 f$ & $3.33 \pm 1.36 \mathrm{de}$ & $150.85 \pm 31.85$ ef & $143.75 \pm 52.29 \mathrm{e}$ & 63. \\
\hline \multirow[t]{3}{*}{$\mathrm{Cu}$} & 50 & $86.66 \pm 2.52 b$ & $0.58 \pm 0.16 \mathrm{~cd}$ & $2.33 \pm 0.47 \mathrm{~cd}$ & $2.94 \pm 0.61 b c$ & $4.20 \pm 0.73 c$ & $254.78 \pm 52.92 b c$ & $181.25 \pm 51.34 \mathrm{~cd}$ & 10 \\
\hline & 100 & $61.00 \pm 3.70 \mathrm{e}$ & $0.38 \pm 0.16 e$ & $1.50 \pm 0.38 f$ & $1.88 \pm 0.46 f$ & $4.55 \pm 2.37 \mathrm{c}$ & $114.68 \pm 28.41 \mathrm{~g}$ & $118.75 \pm 51.34 f$ & 66. \\
\hline & 150 & $76.55 \pm 4.31 d$ & $0.62 \pm 0.13 c$ & $1.58 \pm 0.40 f$ & $2.20 \pm 0.43 \mathrm{~cd}$ & $2.64 \pm 0.90$ ef & $166.65 \pm 32.97 e$ & $193.75 \pm 40.16 b c$ & 70. \\
\hline \multirow[t]{3}{*}{$\mathrm{Fe}$} & 50 & $86.55 \pm 2.42 b$ & $0.42 \pm 0.17 e$ & $1.26 \pm 0.27 \mathrm{~h}$ & $1.86 \pm 0.29 f$ & $3.39 \pm 1.28 \mathrm{~d}$ & $145.58 \pm 25.56 \mathrm{ef}$ & $131.25 \pm 55.90 \mathrm{de}$ & 56. \\
\hline & 100 & $76.66 \pm 4.26 \mathrm{~d}$ & $0.50 \pm 0.14 d$ & $1.84 \pm 0.29 \mathrm{e}$ & $2.34 \pm 0.28 \mathrm{~cd}$ & $3.95 \pm 1.40 \mathrm{~cd}$ & $179.38 \pm 22.08 \mathrm{de}$ & $156.25 \pm 44.19 \mathrm{e}$ & 82. \\
\hline & 150 & $86.66 \pm 2.52 b$ & $0.62 \pm 0.13 c$ & $1.58 \pm 0.50 f$ & $2.20 \pm 0.1 \mathrm{~cd}$ & $2.53 \pm 0.55 \mathrm{ef}$ & $190.65 \pm 53.06 \mathrm{~d}$ & $193.75 \pm 40.74 b c$ & 70. \\
\hline P value & & 0.0011 & 0.0001 & 0.0300 & 0.0005 & 0.0066 & 0.0110 & 0.0009 & 0.0 \\
\hline
\end{tabular}

TABLE 7 Effect of biogenic NPs priming on seed germination parameters of flax on $7^{\text {th }}$ day, under different nanoparticle concentrations. Data are presented as mean values $\pm S D$ for three independent experiments. The same letters within a column show no significant difference at a $95 \%$ probability level at the $p<0.05$ level. 


\begin{tabular}{|c|c|c|c|c|c|c|c|c|}
\hline Nanoparticle & $\begin{array}{l}\text { Concentration } \\
(\mathrm{ppm})\end{array}$ & $\begin{array}{l}\text { Germination } \\
\text { percentage } \\
\text { (G\%) }\end{array}$ & $\begin{array}{l}\text { Shoot } \\
\text { length } \\
(\mathrm{cm})\end{array}$ & $\begin{array}{l}\text { Root length } \\
\text { (cm) }\end{array}$ & $\begin{array}{l}\text { Seedling } \\
\text { length } \\
(\mathrm{cm})\end{array}$ & $\begin{array}{l}\text { Root to } \\
\text { shoot } \\
\text { ratio }\end{array}$ & SVI & SLSI (\%) \\
\hline Non(Control) & 0 & $93.33 \pm 1.87 a$ & $3.91 \pm 0.20 \mathrm{~d}$ & $2.75 \pm 0.68 \mathrm{~h}$ & $6.66 \pm 0.75 h$ & $0.70 \pm 0.16 f$ & $622.20 \pm 70.25 \mathrm{~g}$ & $100.00 \pm 0.00 c$ \\
\hline \multirow[t]{3}{*}{ C-ZnO NPs } & 50 & $86.61 \pm 2.42 b$ & $4.37 \pm 0.62 c$ & $4.50 \pm 0.57 e$ & $8.87 \pm 1.10$ ef & $1.03 \pm 0.11 \mathrm{c}$ & $769.10 \pm 96.07 e$ & $112.17 \pm 16.13 b c$ \\
\hline & 100 & $86.66 \pm 2.52 b$ & $4.42 \pm 0.29 c$ & $5.47 \pm 0.75 d$ & $9.80 \pm 0.62 \mathrm{de}$ & $1.22 \pm 0.23 b$ & $849.26 \pm 54.34 d$ & $115.46 \pm 7.65 b c$ \\
\hline & 150 & $96.66 \pm 1.91 a$ & $4.44 \pm 0.37 \mathrm{c}$ & $6.00 \pm 0.11 \mathrm{~cd}$ & $10.34 \pm 0.65 d$ & $1.33 \pm 0.12 b$ & $999.46 \pm 63.23 b$ & $114.84 \pm 9.69 b c$ \\
\hline \multirow[t]{3}{*}{ NC-Zno NPs } & 50 & $80.23 \pm 2.86 b c$ & $5.37 \pm 0.25 a$ & $7.50 \pm 1.58 b$ & $12.87 \pm 1.37 b$ & $1.40 \pm 0.36 \mathrm{~b}$ & $1032.96 \pm 110.46 \mathrm{ab}$ & $137.82 \pm 6.41 \mathrm{~b}$ \\
\hline & 100 & $80.00 \pm 2.45 b c$ & $4.62 \pm 0.47 \mathrm{bc}$ & $6.00 \pm 0.40 \mathrm{~cd}$ & $10.62 \pm 0.75 d$ & $1.32 \pm 0.13 b$ & $850.00 \pm 60.00 d$ & $118.58 \pm 12.27 b c$ \\
\hline & 150 & $83.33 \pm 3.33 b c$ & $4.12 \pm 0.25 \mathrm{~cd}$ & $4.575 \pm 0.65 \mathrm{e}$ & $8.70 \pm 0.62 f$ & $1.11 \pm 0.18 b c$ & $724.97 \pm 52.26$ ef & $105.76 \pm 6.41 \mathrm{c}$ \\
\hline \multirow[t]{3}{*}{ Zn NPs } & 50 & $86.66 \pm 2.12 b$ & $5.00 \pm 0.22 \mathrm{ab}$ & $8.25 \pm 0.86 a$ & $13.25 \pm 1.25 a$ & $1.64 \pm 0.06 a$ & $1148.24 \pm 109.04 a$ & $128.20 \pm 10.46 a b$ \\
\hline & 100 & $73.35 \pm 3.15 d$ & $5.00 \pm 0.00 \mathrm{ab}$ & $6.37 \pm 1.25 \mathrm{c}$ & $11.37 \pm 1.10 \mathrm{c}$ & $1.28 \pm 0.33 b$ & $834.12 \pm 81.29 d$ & $128.20 \pm 10.46 a b$ \\
\hline & 150 & $73.33 \pm 3.35 d$ & $4.62 \pm 1.18 b c$ & $5.62 \pm 0.62 d$ & $10.22 \pm 0.64 d$ & $1.32 \pm 0.57 \mathrm{~b}$ & $751.63 \pm 47.33 e$ & $118.58 \pm 30.29 b c$ \\
\hline \multirow[t]{3}{*}{ MgO } & 50 & $76.66 \pm 4.26$ & $4.79 \pm 0.50 \mathrm{~b}$ & $4.62 \pm 0.62 e$ & $9.37 \pm 0.75 \mathrm{e}$ & $0.98 \pm 0.17 c$ & $718.68 \pm 57.49$ ef & $121.79 \pm 12.82 b$ \\
\hline & 100 & $80.30 \pm 2.86 \mathrm{bc}$ & $4.37 \pm 0.47 \mathrm{bc}$ & $3.87 \pm 1.10 \mathrm{fg}$ & $8.45 \pm 1.50 f$ & $0.87 \pm 0.17 e$ & $662.47 \pm 120.45 f g$ & $114.17 \pm 12.27 b c$ \\
\hline & 150 & $73.33 \pm 3.25 d$ & $5.00 \pm 0.40 \mathrm{ab}$ & $4.12 \pm 0.75 \mathrm{ef}$ & $9.12 \pm 0.75 \mathrm{e}$ & $0.83 \pm 0.19 e$ & $669.13 \pm 54.99 f$ & $128.20 \pm 10.46 b$ \\
\hline \multirow[t]{3}{*}{$\mathrm{Ag}$} & 50 & $90.05 \pm 1.98 \mathrm{ab}$ & $5.37 \pm 0.62 a$ & $4.87 \pm 0.25 e$ & $10.25 \pm 0.50 \mathrm{~d}$ & $0.91 \pm 0.14 d$ & $923.01 \pm 45.02 c$ & $137.39 \pm 16.13 a$ \\
\hline & 100 & $83.33 \pm 3.33 b c$ & $5.22 \pm 0.63 a$ & $4.75 \pm 1.32 \mathrm{e}$ & $9.97 \pm 1.51 \mathrm{~d}$ & $0.91 \pm 0.26 d$ & $831.21 \pm 126.62 d$ & $133.97 \pm 16.40 \mathrm{a}$ \\
\hline & 150 & $81.00 \pm 2.33 b c$ & $4.85 \pm 1.25 b$ & $4.37 \pm 0.75 e$ & $9.12 \pm 1.84 \mathrm{e}$ & $0.96 \pm 0.25 \mathrm{~cd}$ & $739.12 \pm 149.26 \mathrm{e}$ & $121.79 \pm 32.26 b c$ \\
\hline \multirow[t]{3}{*}{$\mathrm{Cu}$} & 50 & $90.00 \pm 1.93 a b$ & $4.62 \pm 0.47 \mathrm{bc}$ & $5.87 \pm 0.62 c$ & $10.50 \pm 0.91 d$ & $1.27 \pm 0.16 b$ & $945.00 \pm 82.15 b c$ & $118.58 \pm 12.27 b c$ \\
\hline & 100 & $76.26 \pm 4.25 d$ & $3.87 \pm 0.47 d$ & $2.65 \pm 0.47 \mathrm{~h}$ & $6.50 \pm 0.40 \mathrm{~h}$ & $0.69 \pm 0.17 f$ & $498.29 \pm 31.29 \mathrm{i}$ & $99.35 \pm 12.27 \mathrm{~cd}$ \\
\hline & 150 & $76.66 \pm 4.25 d$ & $4.25 \pm 0.28 c$ & $2.77 \pm 0.85 \mathrm{~h}$ & $7.12 \pm 0.62 \mathrm{~g}$ & $0.68 \pm 0.24 f$ & $546.20 \pm 48.23 \mathrm{~h}$ & $108.97 \pm 7.40 \mathrm{c}$ \\
\hline \multirow[t]{3}{*}{$\mathrm{Fe}$} & 50 & $86.56 \pm 2.52 b$ & $3.37 \pm 0.47 e$ & $2.37 \pm 0.62 i$ & $5.75 \pm 1.04 i$ & $0.69 \pm 0.14 f$ & $498.29 \pm 90.19 \mathrm{i}$ & $86.53 \pm 12.27 \mathrm{e}$ \\
\hline & 100 & $76.36 \pm 4.26 \mathrm{~d}$ & $4.12 \pm 0.62 \mathrm{~cd}$ & $2.75 \pm 0.61 \mathrm{~h}$ & $6.87 \pm 0.75 \mathrm{gh}$ & $0.68 \pm 0.21 f$ & $537.03 \pm 57.49 \mathrm{~h}$ & $105.76 \pm 16.13 c$ \\
\hline & 150 & $86.64 \pm 2.42 b$ & $4.12 \pm 0.47 \mathrm{~cd}$ & $2.37 \pm 0.77 i$ & $6.50 \pm 0.00 \mathrm{~h}$ & $0.59 \pm 0.19 g$ & $553.29 \pm 0.00 \mathrm{~h}$ & $105.76 \pm 12.27 \mathrm{c}$ \\
\hline$P$ value & & 0.0000 & 0.0100 & 0.0007 & 0.0001 & 0.0033 & 0.0082 & 0.0004 \\
\hline
\end{tabular}

TABLE 8 Effect of metal salt (precursors) priming on seed germination parameters of flax on $2^{\text {nd }}$ day, under different nanoparticle concentrations. Data are presented as mean values \pm SD for three independent experiments. The same letters within a column show no significant difference at a $95 \%$ probability level at the $p<0.05$ level. 


\begin{tabular}{|c|c|c|c|c|c|c|c|c|c|}
\hline Metal salt & $\begin{array}{l}\text { Concentration } \\
\text { (ppm) }\end{array}$ & $\begin{array}{l}\text { Germination } \\
\text { percentage } \\
\text { (G\%) }\end{array}$ & $\begin{array}{l}\text { Shoot } \\
\text { length } \\
(\mathrm{cm})\end{array}$ & $\begin{array}{l}\text { Root length } \\
\text { (cm) }\end{array}$ & $\begin{array}{l}\text { Seedling } \\
\text { length } \\
(\mathrm{cm})\end{array}$ & $\begin{array}{l}\text { Root to } \\
\text { shoot } \\
\text { ratio }\end{array}$ & SVI & SLSI (\%) & RLSI (\%) \\
\hline Non(Control) & 0 & $86.66 \pm 2.52 b c$ & $0.32 \pm 0.04 d$ & $2.24 \pm 0.28 b$ & $2.56 \pm 0.2 \mathrm{~cd}$ & $7.13 \pm 1.31 a$ & $221 \pm 24 b c$ & $100.00 \pm 0.00 c$ & $100.00 \pm 0$ \\
\hline \multirow[t]{3}{*}{$\mathrm{Zn}\left(\mathrm{CH}_{3} \mathrm{CO}_{2}\right)_{2}$} & 50 & $96.66 \pm 1.91 a$ & $0.52 \pm 0.10 \mathrm{a}$ & $2.62 \pm 0.35 \mathrm{ab}$ & $3.10 \pm 0.3 b$ & $5.28 \pm 1.52 c$ & $303 \pm 31 a$ & $164.50 \pm 34.23 a$ & $116.96 \pm 1$ \\
\hline & 100 & $93.03 \pm 1.87 a b$ & $0.48 \pm 0.08 b$ & $2.56 \pm 0.28 a b$ & $3.04 \pm 0.1 b$ & $5.40 \pm 0.81 \mathrm{c}$ & $283 \pm 30 a b$ & $147.00 \pm 26.14 b$ & $114.28 \pm 1:$ \\
\hline & 150 & $91.00 \pm 1.93 a b$ & $0.42 \pm 0.08 \mathrm{bc}$ & $2.12 \pm 0.88 \mathrm{~b}$ & $2.54 \pm 0.7 \mathrm{~cd}$ & $5.27 \pm 2.15 \mathrm{c}$ & $228 \pm 79 b c$ & $131.25 \pm 26.4 b c$ & $94.64 \pm 39$. \\
\hline \multirow[t]{3}{*}{ MgSO4 } & 50 & $83.33 \pm 3.33 \mathrm{bc}$ & $0.52 \pm 0.04 a$ & $2.52 \pm 0.25 a b$ & $3.04 \pm 0.2 b$ & $4.89 \pm 0.78 d$ & $255 \pm 19 b$ & $164.50 \pm 13.97 a$ & $112.50 \pm 1$ \\
\hline & 100 & $83.33 \pm 2.22 \mathrm{bc}$ & $0.40 \pm 0.12 b c$ & $2.80 \pm 0.57 a$ & $3.2 \pm 0.9 \mathrm{ab}$ & $7.25 \pm 1.49 a$ & $266 \pm 53 b$ & $125.00 \pm 38.2 b c$ & $125.00 \pm 2$ \\
\hline & 150 & $86.66 \pm 2.11 \mathrm{bc}$ & $0.54 \pm 0.16 a$ & $2.72 \pm 0.31 \mathrm{ab}$ & $3.26 \pm 0.35 a$ & $5.58 \pm 2.28 b c$ & $282 \pm 30 a b$ & $168.75 \pm 52.29 a$ & $121.42 \pm 1$ \\
\hline \multirow[t]{3}{*}{ AgNO3 } & 50 & $86.66 \pm 2.52 b c$ & $0.54 \pm 0.15 a$ & $2.88 \pm 0.61 a$ & $3.42 \pm 0.54 a$ & $5.98 \pm 2.96 \mathrm{~b}$ & $297 \pm 46 a$ & $167.75 \pm 47.39 a$ & $128.57 \pm 2$ \\
\hline & 100 & $66.66 \pm 2.54 d$ & $0.26 \pm 0.31 e$ & $0.56 \pm 0.32 \mathrm{ef}$ & $0.82 \pm 0.6 \mathrm{fg}$ & $2.00 \pm 0.88 f$ & $54 \pm 41 f$ & $81.25 \pm 97.82 d$ & $25.00 \pm 14$ \\
\hline & 150 & $0 e$ & of & $0 \mathrm{~g}$ & Oh & $0 \mathrm{~g}$ & $0 \mathrm{~g}$ & of & Oh \\
\hline \multirow[t]{3}{*}{ CuSO4 } & 50 & $86.66 \pm 2.52 b c$ & $0.40 \pm 0.07 b c$ & $1.30 \pm 0.29 d$ & $1.70 \pm 0.29 e$ & $3.34 \pm 0.92 e$ & $147 \pm 25 d$ & $125.00 \pm 22.9 b c$ & $58.03 \pm 13$ \\
\hline & 100 & $93.66 \pm 2.71 \mathrm{ab}$ & $0.22 \pm 0.16 \mathrm{e}$ & $0.70 \pm 0.12 e$ & $0.92 \pm 0.2 f$ & $3.27 \pm 1.88 \mathrm{e}$ & $86 \pm 24 e$ & $68.75 \pm 51.34 \mathrm{e}$ & $31.25 \pm 5.4$ \\
\hline & 150 & $90.33 \pm 3.25 b$ & $0.32 \pm 0.08 d$ & $0.58 \pm 0.16 \mathrm{ef}$ & $0.90 \pm 0.1 f$ & $1.97 \pm 0.76 f$ & $82 \pm 14 e$ & $100.00 \pm 26.14 \mathrm{c}$ & $25.89 \pm 7.3$ \\
\hline \multirow[t]{3}{*}{ FeCl3 } & 50 & $89.66 \pm 1.77 b$ & $0.46 \pm 0.08 b$ & $2.36 \pm 0.35 b$ & $2.82 \pm 0.29 \mathrm{c}$ & $5.46 \pm 2.21 \mathrm{c}$ & $255 \pm 26 b$ & $143.75 \pm 27.95 b$ & $105.35 \pm 1$ \\
\hline & 100 & $91.33 \pm 3.25 \mathrm{ab}$ & $0.38 \pm 0.08 c$ & $1.82 \pm 0.20 \mathrm{c}$ & $2.20 \pm 0.5 \mathrm{~d}$ & $5.03 \pm 1.41 \mathrm{~cd}$ & $198 \pm 14 c$ & $120.75 \pm 6.14 \mathrm{bc}$ & $81.25 \pm 9.1$ \\
\hline & 150 & $87.00 \pm 2.12 b c$ & $0.46 \pm 0.13 b$ & $2.58 \pm 0.29 a b$ & $3.04 \pm 0.4 b$ & $5.86 \pm 1.12 b$ & $264 \pm 36 b$ & $143.85 \pm 41.92 b$ & $115.17 \pm 1$ \\
\hline$P$ value & & 0.0111 & 0.0001 & 0.0008 & 0.0039 & 0.0007 & 0.0004 & 0.0100 & 0.0006 \\
\hline
\end{tabular}

TABLE 9 Effect of metal salt (precursors) priming on seed germination parameters of flax on $7^{\text {th }}$ day, under different nanoparticle concentrations. Data are presented as mean values \pm SD for three independent experiments. The same letters within a column show no significant difference at a $95 \%$ probability level at the $p<0.05$ level.

\begin{tabular}{|c|c|c|c|c|c|c|c|c|c|}
\hline Metal salt & $\begin{array}{l}\text { Concentration } \\
(\mathrm{ppm})\end{array}$ & $\begin{array}{l}\text { Germination } \\
\text { percentage } \\
\text { (G\%) }\end{array}$ & $\begin{array}{l}\text { Shoot } \\
\text { length } \\
\text { (cm) }\end{array}$ & $\begin{array}{l}\text { Root length } \\
\text { (cm) }\end{array}$ & $\begin{array}{l}\text { Seedling } \\
\text { length } \\
(\mathrm{cm})\end{array}$ & $\begin{array}{l}\text { Root to } \\
\text { shoot } \\
\text { ratio }\end{array}$ & SVI & SLSI (\%) & RLSI \\
\hline Non(Control) & 0 & $93.33 \pm 1.87 a$ & $3.91 \pm 0.20 \mathrm{de}$ & $2.75 \pm 0.68 \mathrm{~h}$ & $6.66 \pm 0.75 \mathrm{~g}$ & $0.70 \pm 0.16 e$ & $622 \pm 70 f$ & $100.00 \pm 0.00 d$ & $100 . C$ \\
\hline \multirow[t]{3}{*}{$\mathrm{Zn}\left(\mathrm{CH}_{3} \mathrm{CO}_{2}\right)_{2}$} & 50 & $86.66 \pm 2.52 b$ & $5.87 \pm 0.7 a-c$ & $7.12 \pm 1.43 a$ & $13.00 \pm 1.77 a$ & $1.20 \pm 0.19 b c$ & $112 \pm 154 i$ & $150.64 \pm 12.27 b$ & $259 . C$ \\
\hline & 100 & $86.66 \pm 2.52 b$ & $4.25 \pm 0.50 d$ & $5.50 \pm 0.70 \mathrm{~cd}$ & $9.75 \pm 0.64 d$ & $1.35 \pm 0.26 b$ & $844 \pm 55 \mathrm{de}$ & $108.97 \pm 12.82 d$ & 200.5 \\
\hline & 150 & $80.00 \pm 2.45 b c$ & $4.25 \pm 0.86 d$ & $5.87 \pm 1.10 \mathrm{c}$ & $10.12 \pm 1.43 \mathrm{~cd}$ & $1.43 \pm 0.38 b$ & $810 \pm 114 \mathrm{de}$ & $108.97 \pm 22.20 \mathrm{~d}$ & 213.6 \\
\hline \multirow[t]{3}{*}{ MgSO4 } & 50 & $83.33 \pm 3.33 b c$ & $5.00 \pm 0.31 c$ & $5.81 \pm 1.21 \mathrm{c}$ & $10.81 \pm 1.06 \mathrm{c}$ & $1.17 \pm 0.31 \mathrm{c}$ & $901 \pm 88 \mathrm{c}$ & $128.20 \pm 10.46 c$ & $211 . \mathrm{a}$ \\
\hline & 100 & $83.33 \pm 3.33 \mathrm{bc}$ & $6.75 \pm 3.27 a$ & $6.37 \pm 2.46 b$ & $13.12 \pm 3.77 a$ & $1.11 \pm 0.65 c$ & $1093 \pm 314 a$ & $173.07 \pm 84.06 a$ & $231 . \varepsilon$ \\
\hline & 150 & $90.00 \pm 1.93 a$ & $5.12 \pm 1.25 \mathrm{c}$ & $5.75 \pm 0.50 c$ & $10.87 \pm 0.94 c$ & $1.20 \pm 0.39 b c$ & $978 \pm 85 b c$ & $131.41 \pm 32.05 b c$ & $209 . C$ \\
\hline \multirow[t]{3}{*}{ AgNO3 } & 50 & $86.66 \pm 2.52 b$ & $5.62 \pm 0.47 b c$ & $6.25 \pm 0.64 b$ & $11.87 \pm 0.62 b$ & $1.12 \pm 0.19 \mathrm{c}$ & $1029 \pm 54 b$ & $144.23 \pm 12.27 b$ & 227.2 \\
\hline & 100 & $40.00 \pm 4.65 e$ & $0.97 \pm 0.75 \mathrm{~g}$ & $1.92 \pm 1.86 i$ & $2.90 \pm 2.57 i$ & $1.67 \pm 1.03 a$ & $116 \pm 103 i$ & $25.00 \pm 19.45 \mathrm{~g}$ & $70.0 \mathrm{C}$ \\
\hline & 150 & of & Oh & Ok & $\mathbf{0 j}$ & Oh & $0 \mathbf{j}$ & Oh & $0 i$ \\
\hline \multirow[t]{3}{*}{ CuSO4 } & 50 & $76.66 \pm 4.26 \mathrm{c}$ & $5.00 \pm 0.40 \mathrm{c}$ & $3.50 \pm 0.70 \mathrm{~g}$ & $8.50 \pm 0.70 \mathrm{e}$ & $0.70 \pm 0.17 e$ & $651 \pm 54 f$ & $128.20 \pm 10.46 \mathrm{c}$ & 127.2 \\
\hline & 100 & $73.33 \pm 3.25 \mathrm{~cd}$ & $3.37 \pm 0.85 e$ & $4.62 \pm 0.28 e$ & $4.00 \pm 0.62 h$ & $0.24 \pm 0.14 \mathrm{~g}$ & $302 \pm 46 h$ & $86.53 \pm 21.89 e$ & $27.2 \epsilon$ \\
\hline & 150 & $80.03 \pm 2.86 \mathrm{bc}$ & $3.12 \pm 0.25 \mathrm{ef}$ & $0.75 \pm 0.28 j$ & $3.97 \pm 0.47 \mathrm{~h}$ & $0.23 \pm 0.08 \mathrm{~g}$ & $310 \pm 38 \mathrm{~h}$ & $80.12 \pm 6.41$ ef & $27.2 \overline{1}$ \\
\hline \multirow[t]{3}{*}{$\mathrm{FeCl} 3$} & 50 & $81.00 \pm 2.33 b c$ & $5.75 \pm 0.50 \mathrm{bc}$ & $5.00 \pm 0.70 d$ & $10.75 \pm 1.19 \mathrm{c}$ & $0.86 \pm 0.05 d$ & $870 \pm 96 d$ & $147.43 \pm 12.82 b$ & $181 . \varepsilon$ \\
\hline & 100 & $70.66 \pm 1.33 \mathrm{~cd}$ & $5.25 \pm 0.50 \mathrm{c}$ & $2.87 \pm 1.10 \mathrm{~h}$ & $8.10 \pm 0.47$ ef & $0.58 \pm 0.30 \mathrm{ef}$ & $574 \pm 33 g$ & $134.61 \pm 22.20 \mathrm{bc}$ & 104.5 \\
\hline & 150 & $80.30 \pm 2.86 \mathrm{bc}$ & $6.00 \pm 0.57 a b$ & $4.25 \pm 0.86 \mathrm{ef}$ & $10.25 \pm 0.86 \mathrm{~cd}$ & $0.71 \pm 0.17 e$ & $823 \pm 69 \mathrm{de}$ & $153.84 \pm 14.80 \mathrm{~b}$ & 154.5 \\
\hline$P$ value & & 0.0044 & 0.0400 & 0.0006 & 0.0007 & 0.0055 & 0.0050 & 0.0001 & $0.00 \mathrm{C}$ \\
\hline
\end{tabular}




\section{Effect of biogenic NPs and their counterpart salts on physiological characteristics of wheat seedling}

As seed germination is the first step to start a successful crop improvement, it could be considered as an index to assay the enhancive or inhibitive effect of newly developed agrochemicals such as nanomaterials (Ahmed et al., 2019).

In present study, the seeds were exposed to the NPs or salts only for 12 hours but the priming effect was observed up to several days. In this regard, it could be suggested that the NPs or metal ions are absorbed on the surface of the seeds and gradually release to show their effect during a period of seven days. Also, the reason of difference between response of nano-primed seeds with salt-primed ones may be illustrated as the result of gradually release of ions from NPs by sub-toxic levels rather than the exposure to a large number of ions in the case of priming with metal salts which may cause stress in the germination process (Szollosi et al. 2020).

Previous findings have reported that the zinc nanoparticulate priming were more effective than zinc salt in enhancing the seedling growth. For instance, it is found that $\mathrm{ZnNP}$ treated wheat seeds surpassed elemental $\mathrm{Zn}$ values over $\mathrm{ZnSO}_{4}$, indicating that NPs are more efficient at delivering $\mathrm{Zn}$ to plant tissues than $\mathrm{ZnSO}_{4}$, which suggests it is done during a particle-specific mechanism (Baddar et al. 2018). Similar studies have shown that accumulation of $\mathrm{Zn}$ from NP treatment was more than predicted values upon dissolved Zn concentration (Ahmed et al. 2019). However, $\mathrm{Zn}$ is an essential metal for plant growth, it may be a phytotoxic metal when exceeds the tolerance limit depending on the plant species or plant's studied part (Zaeem et al. 2020).

Previous studies also reported similar results to our findings, on seedling growth of ZnO treated wheat seeds (Rawat et al. 2018; Awasthi et al. 2017). In the study conducted by Ahmed et al., C-ZnO NPs with very high concentrations of $0.05,0.5,2,5 \mathrm{mg} / \mathrm{ml}$ were applied on four different seeds such as radish, cucumber, tomato and alfalfa to study the toxicity effect of NPs on seeds. They reported that C-ZnO exhibited no obvious toxic effect on germination, root and shoot growth of these seeds (Ahmed et al. 2019). Similarly, in our study C-ZnO and NC-ZnO NP treatment improved the seedling growth. Several mechanisms could be found in the literature illustrating various effects of NPs on plant parts and cell reactions. For example, the effect of NPs on specific enzymatic reactions and different enzymes such as amylase could elucidate NPs effect on seed germination. It is not clear at this point whether NPs toxicity is stimulated by particles or the dissolved ions (Ko et al. 2017). The effect of NPs may also be due to the interaction of NPs with some parts of the plants such as cell wall or membrane components. The size of the NPs is consistent with structure of the plant cell wall to enter the cell, at the point that the accumulation of reactive oxygen species (ROS) can be started (Zaeem et al., 2020). ROS can influence the permeability of the cells as it is interfered with the plasma membrane. Consequently, more NPs can result in intense stress after reaching the cells and stimulating the formation of stress-induced secondary metabolites (Zaeem et al. 2020).

In another study, Ag NPs toxicity on rockcress seeds was shown to be dependent on the size and concentration (Szollosi et al. 2020). Ag NPs with size of $80 \mathrm{~nm}$ were only deteriorative at higher concentrations and those of 20 and $40 \mathrm{~nm}$ resulted in severe root growth inhibition. The researchers supposed that Ag NPs apoplastically transported through the root tissues (Szollosi et al. 2020). The inhibitory effect of Ag NPs on the germination index was also seen in the case of cucumber (Szollosi et al. 2020). Similar results are reported by Vanninia et al. (2014) as 10 ppm concentration of Ag NPs influenced the seedling growth of wheat seeds adversely. They also reported induction of morphological modifications in root tip cells by Ag NPs. According to the microscopy of the treated seeds roots, Ag NPs did not enter the root cells and located in the outer cells of the root cup. It was suggested by TEM analysis, that the toxicity effect of $\mathrm{Ag}$ NPs is resulted from release of $\mathrm{Ag}^{+}$ions from Ag NPs (Vanninia et al., 2014). Abbasi Khalaki et al. (2016) reported an enhancement in seedling growth of thymus kotschyanus seeds treated with 20 and $60 \%$ concentrations of Ag NPs.

The results reported by Zakharova et al. (2019) is comparable with our obtained results, in which wheat seeds were soaked in the presence of CuO NPs. They also reported that exposure of wheat seeds to 10ppm CuO NPs showed a $14.5 \%$ improvement in germination and a twofold increase in root and shoot length in comparison with control. At higher concentrations of CuO NPs, both stimulation and toxic effects were observed (decline in root length) (Zakharova et al. 2019).

The effect of wheat seed treatment with Cu NPs on germination and seedling vigor index has been studied by Yasmeen et al. under laboratory conditions (Yasmeen et al. 2015). Germination percentage, root and shoot lengths were calculated and the results indicated exposure of wheat seeds to Cu NPs lead to a decline in germination percentage and severe reduction of root and shoot length. Therefore, Cu NPs adversely affect germination and growth of wheat seeds (Yasmeen et al. 2015). This substantial decrease in the plantlet growth is consistent with previous wheat field studies, where the application of excessive NPs resulted in reduced plantlet length and distorted plantlet physiology (Du et al. 2011).

Plaksenkova et al. (2019) studied the effect of $\mathrm{Fe}_{3} \mathrm{O}_{4}$ NPs stress on the growth and development of rocket seeds. According to the results, $1 \mathrm{ppm}$, $2 \mathrm{ppm}$, and 4 ppm concentrations of $\mathrm{Fe}_{3} \mathrm{O}_{4} \mathrm{NPs}$ have positive effect on the growth and development of rocket seedlings. In a similar research, Yi Hao et al. studied the effects nano-priming with different $\mathrm{Fe}_{2} \mathrm{O}_{3}$ morphologies like $\mathrm{Fe}_{2} \mathrm{O}_{3}$ nanocubes, $\mathrm{Fe}_{2} \mathrm{O}_{3}$ short nanorods, $\mathrm{Fe}_{2} \mathrm{O}_{3}$ long nanorods at the concentrations of 5 to 150 ppm on rice seeds during the germination. They found that all NPs considerably stimulated the root growth, and promoted shoot length at most concentrations while $\mathrm{Fe}_{2} \mathrm{O}_{3}$ long nanorods inhibited the seeds germination significantly and showed different biological effect from other Fe $\mathrm{O}_{3}$ nanomaterials, due to their different shape (Hao et al. 2016).

Ngo et al. (2014) studied the effects of Fe NPs on soybean germination, growth, crop yield and product quality. In laboratory conditions, the germination rates of soybean seeds soaked with Fe NPs was $80 \%$ at the concentration of $0.08 \mathrm{~g} / \mathrm{ha}$, while germination of the control sample was $55 \%$. Consequently, various data obtained from such experiments could be really helpful in developing priming treatments for field experiments and other agricultural purposes.

Effect of biogenic NPs and their counterpart salts on physiological characteristics of flax seedling 
The special structure of flax seed's coat was the reason we chose this plant for our experiments. The envelope or testa of the flax seed contain about $15 \%$ of mucilage, that mainly contains distinct types of arabinoxylans and water-soluble hydrocolloid/polysaccharides, which contribute to its gel qualities by forming large aggregates in solution (Mehtre et al. 2017). It is suggested that nano-priming of the flax seeds and also the NPs behavior may be affected by mucilage of the seed coat due to the thick chemical environment in which NPs were trapped. Therefore, according to the obtained results, it could be considered as one the reasons that we observed different results of flax seeds seedling in comparison with wheat seeds.

There are a few works studying the influence of NPs on seedling parameters of flax seeds. In support of our results, the effect of different metal and metal oxide NPs have been presented. As an example, it was reported that the application of biosynthesized MgO NPs enhanced the seed germination and growth parameters of peanut seeds as compared with control. The authors by using physicochemical methods including UV and SEM analyses, indicated that the MgO NPs penetrates into the seed coat, support water uptake inside the seeds, and then affect seed germination and growth rate mechanism (Jhansi et al. 2017).

In contrast of the results obtained from our research, Gorczyca et al. noticed that the 100ppm of Ag NP treatments applied to flax seeds had a limited effect on the germination and early development of the seedlings in comparison with the control. The response of the flax seeds to the NPs was reported as an increase of chlorophyll content (Gorczyca et al. 2018). Zaeem et al. (2020) investigated the effect of green synthesized C-ZnO NPs at concentrations of $0,1,10,100$, 500 and $1000 \mathrm{ppm}$ on growth of flax seeds. All the treated flax seeds had root development of different lengths, ranging from $2.62 \mathrm{~cm}$ (for $1000 \mathrm{ppm} \mathrm{ZnO} \mathrm{NPs)}$ to $7.08 \mathrm{~cm}$ (for $10 \mathrm{ppm} \mathrm{ZnO} N P s$ ) with $3.85 \mathrm{~cm}$ for the control. These results indicate efficiency of different concentrations of ZnO NPs in seed germination. At ZnO NPs concentration of above 10ppm, the higher the concentration of NPs, the lower the root length. The increased sensitivity of radicle to NPs is due to the large surface area of the NPs. They suggested that the observed inhibitory effect on seed germination may be because of the very small size of NPs and the dissolution power of $\mathrm{ZnO}$ to $\mathrm{Zn}^{2+}$ ions (Zaeem et al. 2020).

To the best of our knowledge, there was not any report on positive or negative effect of Cu NPs on flax seedling to be compare with our findings. In previously reported researches, despite our findings, stimulating effects of Fe NPs on seedling of different species have been described, for example in rocket (Ko et al. 2017), rice (Hao et al. 2016) and soybean (Ngo et al. 2014). Clearly, different results obtained from flax seed priming in comparison to the wheat seeds in germination and seedling growth parameters.

\section{A comparison between the effect of biogenic NPs and their counterpart salts on physiological characteristics of wheat and flax seedlings}

At $7^{\text {th }}$ day of the experiment, in a comparison between the effect of studied NPs in applied concentrations, the most effective one in shoot and root development was Zn NP and the less effective NPs were Ag for wheat and Fe for flax seeds (Fig. 2). It shows Zn NPs are not toxic at the applied concentrations and even show stimulating effect on both wheat and flax seeds. Vice versa the applied concentrations of Ag NPs are toxic for wheat but stimulating for flax. As all of the experiment conditions are the same for all of the samples, it could be concluded these differences are related to the seed species. Among the tested metal slats, zinc acetate had the most stimulating effect and $\mathrm{CuSO}_{4}$ was the most toxic one for both flax, and wheat. Nano forms of metals and metal oxides, have been reported to significantly improve root or shoot elongation and seed germination of wheat in comparison with bulk materials (Feizi et al. 2012). This kind of growth development mainly depends on the concentration of NP, duration of nano-priming, growth medium and species of plant (Ahmed et al. 2019).

Among measured parameters, root length is more sensitive than shoot length. Between wheat and flax roots, the flax root length was more sensitive against NP and salt treatments and wheat shoot length was the less sensitive parameter. All over, flax seeds were more sensitive to the treatments compared to the wheat seeds. Although the factors which impact the root and shoot elongation following NP exposure are not clear yet, it could be suggested that the polymeric network of flax seeds mucilage trap NPs or metal ions and then the accessibility of them for flax seeds differs from wheat seeds in the period of our experiment (Ahmed et al. 2019).

Figure 3 provides an image for better comparison between germination percentage variations with changes in concentrations of used NPs and salts. In majority of the cases wheat seeds had more $\mathrm{G} \%$ than flax seeds. 150ppmof $\mathrm{AgNO}_{3}$ solution had such a toxic effect on flax seeds that no germination was observed in this treatment. Similarly, metal-based NPs have been reported to show dual impacts on plants growth, such as seed germination. Positive effects of metal-based NPs treatments were displayed in different plants (Szollosi et al. 2020). Seed germination of soybean seeds enhanced by nano-priming with Co, Fe and Cu NPs (Mehtre et al. 2017), also similar findings were reported in the case of some Solanaceae crops after treatment with $\mathrm{ZnO}$ and TiO ${ }_{2} \mathrm{NPs}$ (Younes et al. 2020). The obtained results were comparable with those reported by Feizi et al. (Feizi et al., 2012), in which seed treatment with TiO ${ }_{2} \mathrm{NPs}_{\mathrm{S}}$ at low concentrations (1-2ppm) resulted in an improve in germination of wheat seeds and also seedling elongation compared to untreated wheat, but no significant effect at concentration of 100ppm was observed (Feizi et al., 2012).

\section{Conclusion}

Suggesting that using green synthesized nano-size minerals for seed treatment could be helpful for seedling growth improvement. Present study provides new information on possible positive or toxic effects of seed priming with NPs on germination percentages, shoot length, root length, seedlings length, root/shoot ratio, seedling vigor index (SVI), shoot length stress tolerance index (SLSI) and root length stress tolerance index (RLSI) which were calculated at $2^{\text {nd }}$ and $7^{\text {th }}$ days on two popular early growth plants, wheat and flax. Plant's dual responses varied among NPs type and correlated to the tested concentrations. According to the obtained results, the response of the tested plants to a certain NP was different between flax and wheat. Moreover, it differed between applied concentrations of the NPs. For example, Ag NPs showed a significant positive effect on root and shoot elongation of flax seedlings but there were a dose dependent decrease of root and shoot elongation of wheat seedlings over their respective controls. Another important result is that flax seed was more sensitive to priming with metal salts and NPs in comparison with wheat seed. Furthermore, the influence of these treatments was investigated in earlier stages 
of the growth, in $2^{\text {nd }}$ day of the experiment, in comparison with $7^{\text {th }}$ day of the experiment. Among the studied NPs, Zn and Ag NPs exhibited the best biological effects on growth and development of wheat and flax respectively. The effect of the nanoparticle's counterpart metal salts on seedling parameters also studied for comparison with nanoparticulate ones. Over all, nanoparticle treatments were more effective than metal salt treatments in root and shoot development. The basic mechanisms need to be investigated in future investigations.

\section{Declarations}

\section{Author contribution}

M. B. conducted the majority of the experiments, performed results interpretation as well as manuscript writing, performed experiments; M.Z. performed statistical analysis, contributed to results interpretation and reviewed the manuscript, contributed to the overall design of the experiments, results interpretation, manuscript writing, and reviewed the manuscript. Both authors read and approved the final manuscript.

\section{Supplementary Information}

The online version contains supplementary material available at doi.org/10.3390/

molecules26175402 and doi.org/10.3390/molecules26103025.

Acknowledgments: This paper has been supported by the RUDN University Strategic Academic Leadership Program.

\section{Availability of data and materials}

The datasets used and/or analyzed during the current study are available from the corresponding author on reasonable request.

\section{Declarations}

Ethics approval and consent to participate Not applicable.

\section{Consent for publication}

Not applicable.

\section{Competing interests}

The authors declare no competing interests.

ORCID

Maryam Bayat https://orcid.org/0000-0003-0432-3598

Meisam Zargarhttps://orcid.org/0000-0002-5208-0861

\section{References}

Abbasi Khalaki M, Ghorbani A, Moameri, M (2016) Effects of Silica and Silver Nanoparticles on Seed Germination Traits of Thymus kotschyanus in Laboratory Conditions. Journal of Rangeland Science 6 (3): 221-231.

Ahmed B, Rizvi A, Zaidi A, Khan MS, Musarrat J (2019) Understanding the phyto-interaction of heavy metal oxide bulk and nanoparticles: evaluation of seed germination, growth, bioaccumulation, and metallothionein production. RSC Adv 9, 4210-4225. DOI: 10.1039/c8ra09305a

Alam MJ, Sultana F, Iqbal MT (2015) Potential of Iron Nanoparticles to Increase Germination and Growth of Wheat Seedling. J Nanosci Adv Tech 13:14-20. doi: https://doi.org/10.24218/jnat.2015.12

Al-Hakkani MF (2020) Biogenic copper nanoparticles and their applications: A review. SN Applied Sciences 2, 505-525. https://doi.org/10.1007/s42452-0202279-1

Allaby M (2006) A Dictionary of Plant Sciences (2 ed.), Oxford University Press.

Asanova AA, Yashin SE, Trofimova TV, Polonskiy VI (2019) Application of silver nanoparticles to improve wheat seedlings growth, IOP Conf. Series: Earth and Environmental Science 315:052041-6, doi:10.1088/1755-1315/315/5/052041

Aslani F, Bagheri S, Julkapli N, Juraimi AS, Golestan Hashemi FS, Baghdadi A (2014) Effects of Engineered Nanomaterials on Plants Growth: An Overview. The Scientific World Journal 28 p, http://dx.doi.org/10.1155/2014/641759

Awasthi A, Bansal S, Jangir LJ, Awasthi G, Awasthi KK, Awasthi K (2017) Effect of ZnO Nanoparticles on Germination of Triticum aestivum Seeds. Macromol. Symp 376: 1700043-8. https://doi.org/10.1002/masy.201700043 
Baddar ZE, Unrine JM (2018) Functionalized-ZnO-Nanoparticle Seed Treatments to Enhance Growth and Zn Content of Wheat (Triticum aestivum) Seedlings. J. Agric. Food Chem 66: 12166-12178, DOI: 10.1021/acs.jafc.8b03277

Bayat M, Chudinova E, Zargar M, Lyashko M, Louis K, Adenew K (2019) Phyto-assisted green synthesis of zinc oxide nanoparticles and its antibacterial and antifungal activity. Research on Crops 20 (4): 725-730. DOI : 10.31830/2348-7542.2019.107

Bayat M, Zargar M, Astarkhanova T, Pakina E, Ladan S, Lyashko M, Shkurkin SI (2021a) Facile Biogenic Synthesis and Characterization of Seven Metal-Based Nanoparticles Conjugated with Phytochemical Bioactives Using Fragaria ananassa Leaf Extract. Molecules 26(10): 3025-

3049. https://doi.org/10.3390/molecules26103025

Bayat M, Zargar M, Chudinova E, Astarkhanova T, Pakina E (2021b) In Vitro Evaluation of Antibacterial and Antifungal Activity of Biogenic Silver and Copper Nanoparticles: The First Report of Applying Biogenic Nanoparticles against Pilidium concavum and Pestalotia sp. Fungi. Molecules 26: 54025413. https://doi.org/10.3390/molecules26175402

Du W, Sun Y, Ji R, Zhu J, Wu J, Guo H (2011) $\mathrm{TiO}_{2}$ and C-ZnO nanoparticles negatively affect wheat growth and soil enzyme activities in agricultural soil. Journal of Environmental Monitoring 13: 822-828. DOI:10.1039/c0em00611d

Feizi H, Rezvani Moghaddam P, Shahtahmassebi N, Fotovat A (2012) Impact of bulk and nanosized titanium dioxide $\left(\mathrm{TiO}_{2}\right)$ on wheat seed germination and seedling growth. Biol. Trace Elem. Res 146(1): 101-107. DOI: 10.1007/s12011-011-9222-7

Gorczyca A, Przemieniecki SW, Kurowski T, Oćwieja M (2018) Early plant growth and bacterial community in rhizoplane of wheat and flax exposed to silver and titanium dioxide nanoparticles. Environmental Science and Pollution Research 25: 33820-33826. https://doi.org/10.1007/s11356-018-3346-7

Hao Y, Zhang Z, Rui Y, Ren J, Hou T, Wu S, Rui M, Jiang F, Liu L (2016) Effect of Different Nanoparticles on Seed Germination and Seedling Growth in Rice, $2^{\text {nd }}$ Annual International Conference on Advanced Material Engineering (AME 2016), 166-173. DOI:10.2991/ame-16.2016.28

Jhansi K, Jayarambabu N, Reddy KP, Manohar Reddy NM, Suvarna RP, Rao KV, Kumar VR, Rajendar V (2017) Biosynthesis of MgO nanoparticles using mushroom extract: effect on peanut (Arachis hypogaea L.) seed germination. 3 Biotech 7: 263-274. D0I 10.1007/s13205-017-0894-3

Ko KS, Koh DC, Kong IC (2017) Evaluation of the Effects of Nanoparticle Mixtures on Brassica Seed Germination and Bacterial Bioluminescence Activity Based on the Theory of Probability. Nanomaterials 7: 344-344. doi:10.3390

Liu R, Lal R (2014) Synthetic apatite nanoparticles as a phosphorus fertilizer for soybean (Glycine max). Scientific reports. 4: 5686, 6 p. DOI: $10.1038 /$ srep05686

Mehtre AS, Syed HM, Agrawal RS (2017) Extraction and chemical composition of flaxseed gum (mucilage) from different flaxseed varieties. The Bioscan 12(1): 47-49.

Ngo QB, Dao TH, Nguyen HC, Tran XT, Nguyen TV, Khuu TD, Huynh TH (2014) Effects of nanocrystalline powders (Fe, Co and Cu) on the germination, growth, crop yield and product quality of soybean (Vietnamese species DT-51). Advances in Natural Sciences: Nanoscience and Nanotechnology 5: 015016-015023. doi:10.1088/2043-6262/5/1/015016

Plaksenkova I, Jermalınoka M, Bankovska L, Gavarāne I, Gerbreders V, Sledevskis E, Sniḳeris J, Kokina I (2019) Effects of Fe304 Nanoparticle Stress on the Growth and Development of Rocket Eruca sativa. Journal of Nanomaterials 4: 1-10. https://doi.org/10.1155/2019/2678247

Rai M, Shegokar S (2017) Metal Nanoparticles in Pharma. Chapter 15: Antimicrobial Activities of Metal Nanoparticles, Springer International Publishing. ISBN 13: 9783319637907

Raskar S, Laware SL (2013) Effect of titanium dioxide nano particles on seed germination and germination indices in onion. Plant Sciences Feed 3 (9): 103107.

Rastogi A, Zivcak M, Sytar O, Kalaji HM, He X, Mbarki S, Brestic M (2017) Impact of Metal and Metal Oxide Nanoparticles on Plant: A Critical Review. Frontiers in Chemistry 5: 78, 16p. doi: 10.3389/fchem.2017.00078

Rawat PS, Kumar R, Ram P, Pandey P (2018) Effect of Nanoparticles on Wheat Seed Germination and Seedling Growth. International Journal of Agricultural and Biosystems Engineering 12(1): 13-16. doi.org/10.5281/zenodo.1315657

Salah SM, Yajing G, Dongdong C, Jie L, Aamir N, Qijuan H, Weimin H, Mingyu N, Jin H (2015) Seed priming with polyethylene glycol regulating the physiological and molecular mechanism in rice (Oryza sativa L.) under nano-ZnO stress. Scientific Reports 5: 14278, 14 p. DOI: 10.1038/srep14278

Singh A, Singh NB, Afzal S, Singh T, Hussain I (2018) Zinc oxide nanoparticles: a review of their biological synthesis, antimicrobial activity, uptake, translocation and biotransformation in plants. J Mater Sci 53: 185-201. DOI 10.1007/s10853-017-1544-1

Szollosi R, Molnár A, Kondak S, Kolbert Z (2020) Dual Effect of Nanomaterials on Germination and Seedling Growth: Stimulation vs. Phytotoxicity. Plants 9: 1745-1776. doi:10.3390/plants9121745

USEPA (1996) United States Environmental Protection Agency- USEPA. Ecological effects test guidelines terrestrial plant toxicity, tier I (seedling emergence). 
Ushahra J, Malik CP (2013) Ascorbic acid mediated enhancement in growth and antioxidant status of Eruca sativa varieties. Tech Journal of Biotechnology 2 (4): 53-64. http://www.cibtech.org/cjb.htm

Vanninia C, Domingoa G, Onellib E, De Mattiac F, Brunic I, Marsonia M, Bracale M (2014) Phytotoxic and genotoxic effects of silver nanoparticles exposure on germinating wheat seedlings. Journal of Plant Physiology 171: 1142-1148. DOI:10.1016/j.jplph.2014.05.002

Yang J, Cao W, Rui Y (2017) Interactions between nanoparticles and plants: phytotoxicity and defense mechanisms. Journal of Plant Interactions 12(1): 158169. DOI: $10.1080 / 17429145.2017 .1310944$

Yasmeen F, Razzaq A, lqbal MN Jhanzab HM (2015) Effect of silver, copper and iron nanoparticles on wheat germination. International Journal of Biosciences. 6(4), 112-117. DOI:10.12692/ijb/6.4.112-117

Younes NA, Hassan HS, Elkady MF, Hamed AM, Dawood MF (2020) Impact of synthesized metal oxide nanomaterials on seedlings production of three Solanaceae crops. Heliyon 6: 3188-3198. https://doi.org/10.1016/j.heliyon.2020.e03188

Zaeem A, Drouet S, Anjum S, Khurshid R, Younas M, Blondeau JP, Tungmunnithum D, Giglioli-Guivarc'h N, Hano C, Abbasi BH (2020) Effects of biogenic zinc oxide nanoparticles on growth and oxidative stress response in flax seedlings vs. In vitro cultures: a comparative analysis. Biomolecules 10: $918-934$.

doi:10.3390/biom10060918

Zakharova OV, Kolesnikov EA, Shatrova N, Gusev A (2019) The effects of CuO nanoparticles on wheat seeds and seedlings and Alternaria solani fungi: in vitro study, FORESTRY, IOP Conf. Series: Earth and Environmental Science 226: 012036-012046. doi:10.1088/1755-1315/226/1/012036

Zhao X, Joo JC, Kim D, Lee JK, Kim JY (2016) Estimation of the Seedling Vigor Index of Sunflowers Treated with Various Heavy Metals. J Bioremed Biodeg 7: 353-359. doi:10.4172/2155-6199.1000353

\section{Figures}

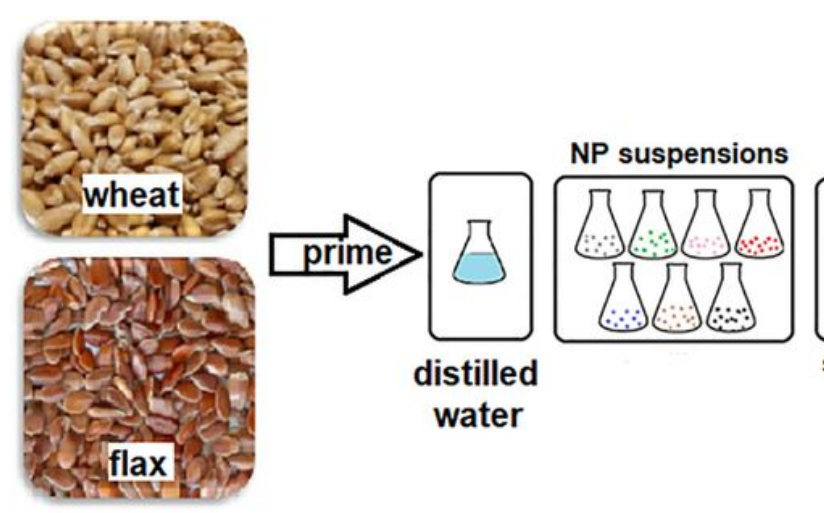

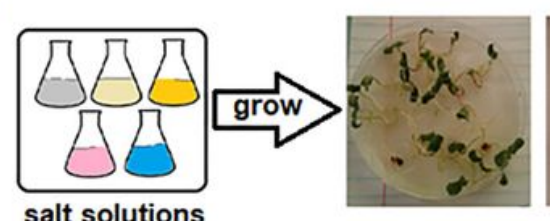

salt solutions
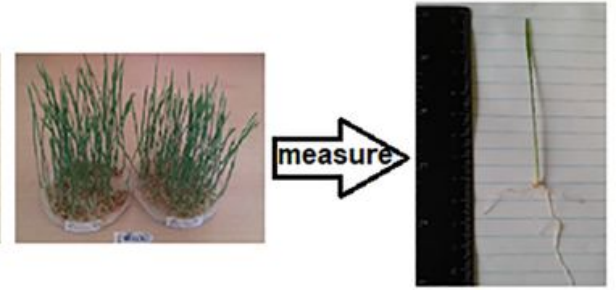

Figure 1

Graphical summary of the experiment 


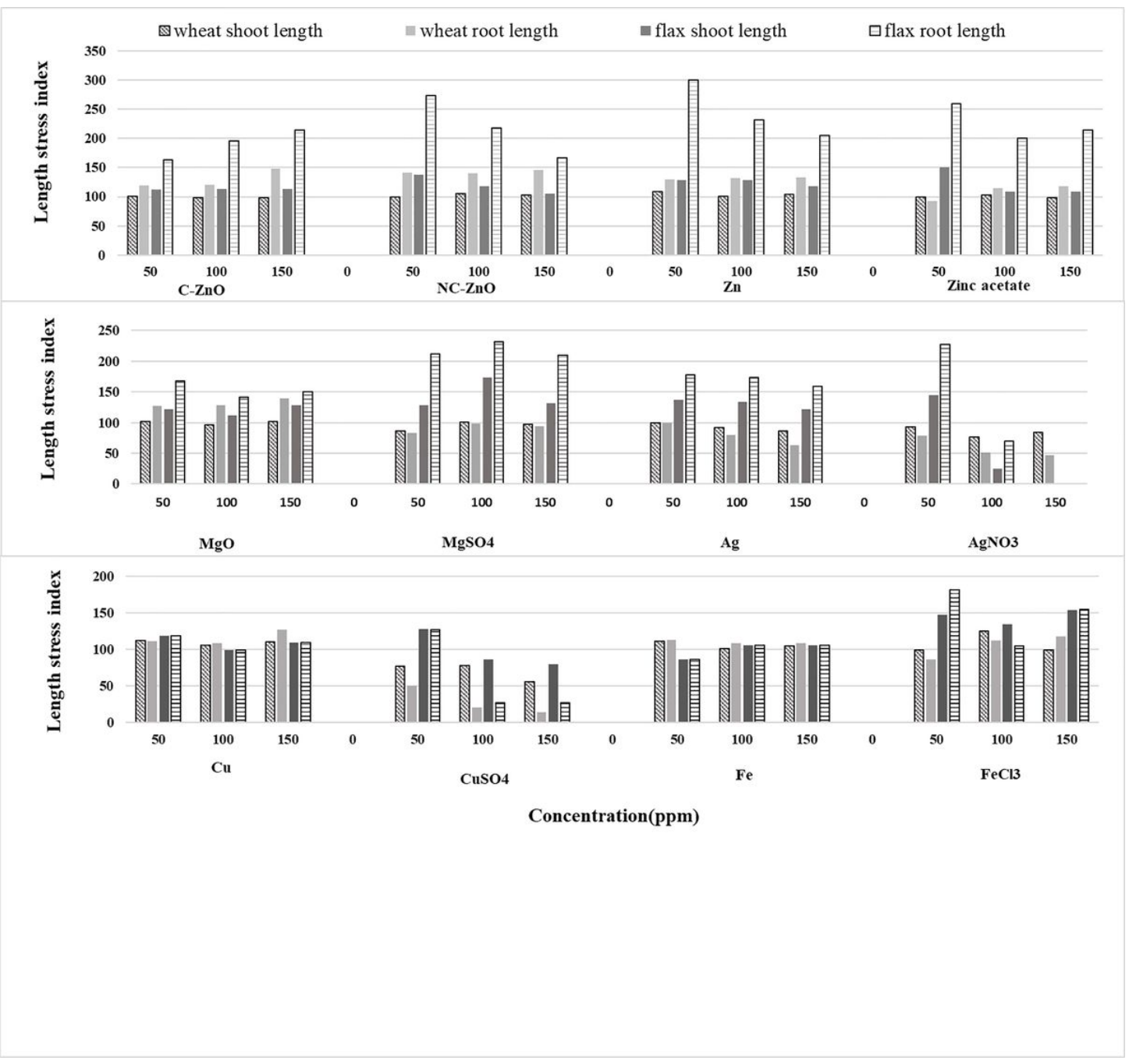

Figure 2

Dose response effect of NPs and their correspondent metal salts on shoot and root stress tolerance index (SLSI and RLSI, respectively) of wheat and flax seeds at 7th day of the experiment. 


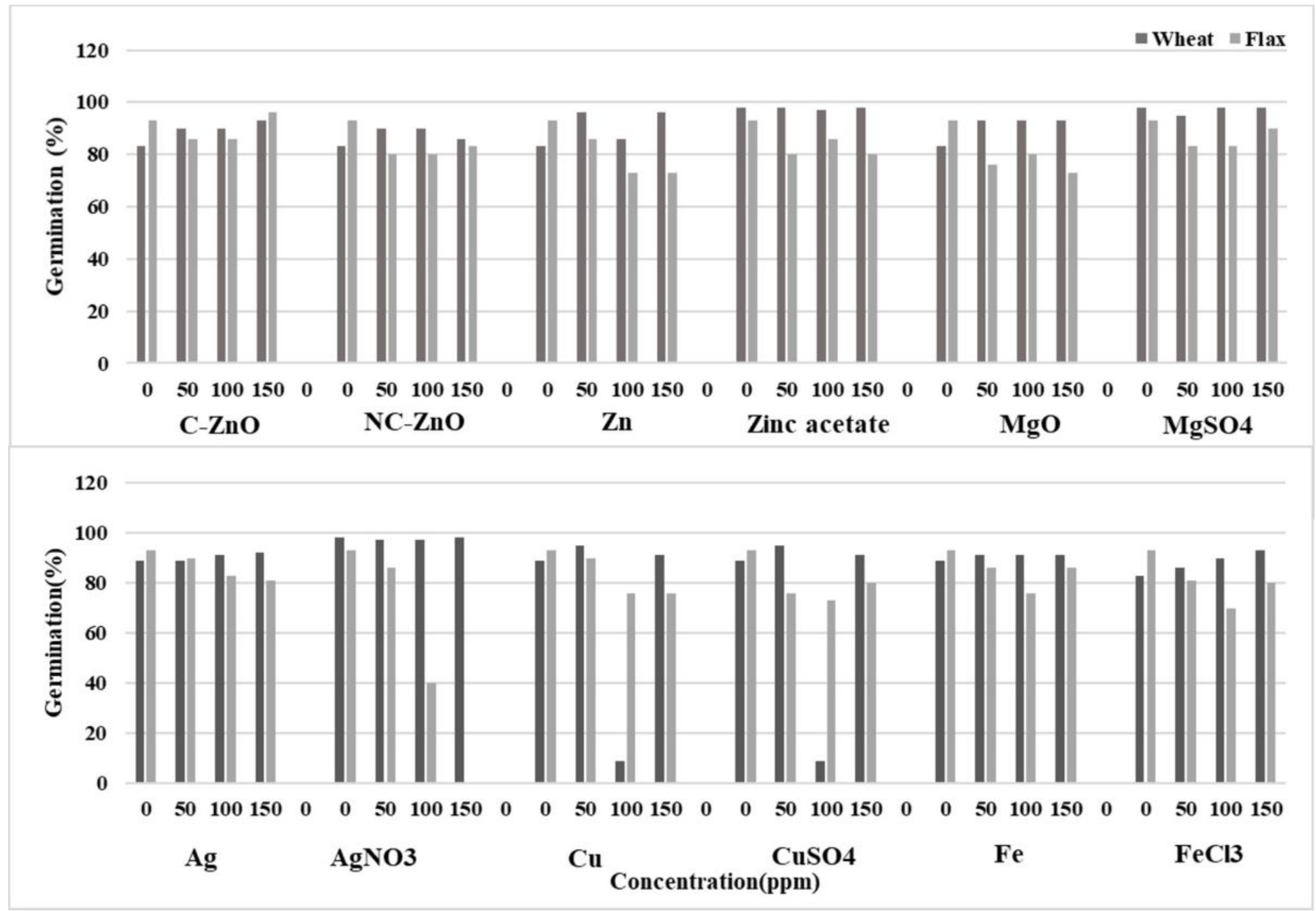

Figure 3

Dose response effect of NPs and their correspondent metal salts on germination percentage of wheat and flax seeds at 7th day of the experiment. 